Proceedings of Budapest School and Conference

Nonperturbative Quantum Field Theoretic Methods and their Applications, August 2000,

(c) World Scientific Publishing Company

\title{
CONFORMAL BOUNDARY CONDITIONS and what they teach us
}

\author{
VALENTINA B. PETKOVA \\ Institute for Nuclear Research and Nuclear Energy \\ 72 Tzarigradsko Chaussee, 1784 Sofia, Bulgaria,

\begin{abstract}
The question of boundary conditions in conformal field theories is discussed, in the light of recent progress. Two kinds of boundary conditions are examined, along open boundaries of the system, or along closed curves or "seams". Solving consistency conditions known as Cardy equation is shown to amount to the algebraic problem of finding integer valued representations of (one or two copies of) the fusion algebra. Graphs encode these boundary conditions in a natural way, but are also relevant in several aspects of physics "in the bulk". Quantum algebras attached to these graphs contain information on structure constants of the operator algebra, on the Boltzmann weights of the corresponding integrable lattice models etc. Thus the study of boundary conditions in Conformal Field Theory offers a new perspective on several old physical problems and offers an explicit realisation of recent mathematical concepts.
\end{abstract}

\section{Introduction}

The study of boundary conditions in conformal field theories (CFT) and in the related integrable models has been experiencing a renewal of interest over the last three or four years. This has been caused by its relevance in string and brane theory on the one hand, and in various problems of condensed matter on the other: see the lectures of C. Schweigert at this school for an introduction and references to the first subject, and [1] for the second. As a result, there has been a blossoming of papers studying the possible boundary conditions, the boundary fields and their couplings in the framework of CFT 
(see [2] for a fairly extensive bibliography as of mid 99); a systematic discussion of boundary conditions preserving the integrability, both in lattice models [3] and in (classical or quantum) field theories [4]; and an investigation of what happens to a critical system in the presence of boundary perturbations, its renormalisation group flows, etc: see in particular the lectures by G. Watts at this school and [5]. At the same time, new and unexpected connections with "pure" mathematics -operator algebras, quantum symmetries- have also been revealed.

The purpose of these lectures is of course not to present exhaustively all these interesting developments, but just to offer a pedagogical (and maybe somewhat biaised) introduction to their simplest aspects and to some of the recent progress. After briefly recalling basic facts on CFT, their chiral constituents and how they are assembled into physically sensible theories, we turn to the discussion of boundary conditions. We show how solving the consistency condition known as Cardy equation amounts to the algebraic problem of finding non negative integer valued matrix representations of the fusion algebra. These matrices are the adjacency matrices of graphs, which thus encode the boundary conditions (b.c.) in a natural way (sect. 2). The study of the operator algebra of boundary fields (sect. 3) and of possible twisted b.c. (sect. 4) exposes new algebraic features attached to these graphs (sect. 5). The latter also contain information on Boltzmann weights of associated lattice integrable models, as we mention briefly in section 6 .

\section{A lightning review of CFT}

This section is devoted to a fast summary of concepts and notations in rational conformal field theories (RCFT).

\subsection{Chiral data of RCFT}

A rational conformal field theory is defined in terms of a certain number of data. The first set of data specifies the properties of each chiral half, i.e. of the holomorphic or of the antiholomorphic sector of the theory. One is given a certain chiral algebra, $\mathfrak{A}$ : it may be the Virasoro algebra Vir itself, with its generators $L_{n}, n \in \mathbb{Z}$, or equivalently the energy-momentum tensor $T(z)=\sum_{n \in \mathbb{Z}} z^{-n-2} L_{n}$. It may also be one of the extensions of Vir: superconformal algebra, current algebra, $W$-algebra etc. One is also given a finite set $\mathcal{I}$ of irreducible representation spaces (modules) $\left\{\mathcal{V}_{i}\right\}_{i \in \mathcal{I}}$ of $\mathfrak{A}$. Each of these representations of $\mathfrak{A}$ is also a representation (reducible or irreducible) of Vir, 
with a central charge $c$ and with a conformal weight (the lowest eigenvalue of $L_{0}$ ) denoted $h_{i}$. Let's recall for future use that $c$ also specifies the coefficient of the anomalous term in the transformation of the energy-momentum tensor $T(z)$ under an analytic change of coordinate $z \mapsto \zeta(z)$

$$
\tilde{T}(\zeta)=\left(\frac{\partial z}{\partial \zeta}\right)^{2} T(z)+\frac{c}{12}\{z, \zeta\}
$$

where $\{z, \zeta\}$ denotes the schwarzian derivative

$$
\{z, \zeta\}=\frac{\frac{\partial^{3} z}{\partial \zeta^{3}}}{\frac{\partial z}{\partial \zeta}}-\frac{3}{2}\left(\frac{\frac{\partial^{2} z}{\partial \zeta^{2}}}{\frac{\partial z}{\partial \zeta}}\right)^{2} .
$$

By convention, the label $i=1$ denotes the identity representation (for which $\left.h_{1}=0\right)$. Finally, we denote by $\mathcal{V}_{i^{*}}$ the complex conjugate representation of $\mathcal{V}_{i}$; the identity representation is self-conjugate, $1^{*}=1$.

Each of these representations is graded for the action of the Virasoro generator $L_{0}$ : all the eigenvalues of $L_{0}$ differ from the lowest one, $h_{i}$, by a non negative integer ${ }^{1}$, and the eigenspace of eigenvalue $h_{i}+p$ has a certain dimension $\#_{p}^{(i)}$. It is natural to introduce the "character" of the representation $\mathcal{V}_{i}$, which is, up to a prefactor, the generating function of these dimensions

$$
\chi_{i}(q)=\operatorname{tr} q^{L_{0}-\frac{c}{24}}=q^{h_{i}-\frac{c}{24}} \sum_{p=0}^{\infty} \#_{p}^{(i)} q^{p} .
$$

The simplest example is given by the integrable representations of the affine (current) algebra $\widehat{s l}(2)$. For an integer value of the central charge of the affine algebra (or level) $k$, the Virasoro central charge is $c=3 k /(k+2)$, and there is a finite set of integrable representations, labelled by an integer $j: 1 \leq j \leq k+1$, whose conformal weights are $h_{j}=\left(j^{2}-1\right) / 4(k+2)$. In that case, the conjugation is trivial: $\mathcal{V}_{j}=\mathcal{V}_{j^{*}}$. For the representation $(j, k)$, the character reads

$$
\chi_{j}(q)=\frac{1}{\eta^{3}(q)} \sum_{p=-\infty}^{\infty}(2(k+2) p+j) q^{\frac{(2(k+2) p+j)^{2}}{4(k+2)}},
$$

where $\eta(q)=q^{\frac{1}{24}} \prod_{1}^{\infty}\left(1-q^{n}\right)$ is the Dedekind eta function. Such characters are called "specialized characters" since they count states according to their $L_{0}$ grading only. Nonspecialized characters can be introduced, which are sensitive to the Cartan algebra generator $J_{0}$

$$
\chi_{j}(q, u)=\operatorname{tr} q^{L_{0}-\frac{c}{24}} e^{2 \pi i u J_{0}} .
$$

1 This is strictly true only for algebras, whose generators are integrally graded. In a superconformal algebra, or in a parafermionic algebra, the grading would be fractional. 
The expressions of non-specialized characters and/or for higher rank algebras may be found in [6].

Another example is provided by the minimal $c<1$ theories. They are parametrized by a pair of coprime integers $p$ and $p^{\prime}$, and the central charge takes the values $c=1-6\left(p-p^{\prime}\right)^{2} / p p^{\prime}$. The irreducible representations of Vir are labelled by a pair of integers $(r, s), 1 \leq r \leq p^{\prime}-1$, $1 \leq s \leq p-1$, modulo the identification $(r, s) \equiv\left(p^{\prime}-r, p-s\right)$. Their conformal weights read

$$
h_{(r, s)}=h_{\left(p^{\prime}-r, p-s\right)}=\frac{\left(r p-s p^{\prime}\right)^{2}-\left(p-p^{\prime}\right)^{2}}{4 p p^{\prime}} .
$$

Again the conjugation acts trivially. The character of this irreducible representation reads, with the notations $\lambda:=\left(r p-s p^{\prime}\right), \lambda^{\prime}:=\left(r p+s p^{\prime}\right)$

$$
\chi_{(r, s)}(q)=\frac{1}{\eta(q)} \sum_{n \in \mathbb{Z}}\left(q^{\frac{\left(2 n p p^{\prime}+\lambda\right)^{2}}{4 p p^{\prime}}}-q^{\frac{\left(2 n p p^{\prime}+\lambda^{\prime}\right)^{2}}{4 p p^{\prime}}}\right) .
$$

\section{Modular transformations}

In the previous expressions, $q$ is a dummy variable. If, however, $q$ is regarded as a complex variable of modulus less than one and written as $q=\exp 2 i \pi \tau$, with $\tau$ a complex number of positive imaginary part, one may prove that the sum converges and has remarkable modular properties. Under a $\operatorname{PSL}(2, \mathbb{Z})$ transformation of $\tau$ :

$$
\tau \mapsto \frac{a+b \tau}{c+d \tau}, \quad a, b, c, d \in \mathbb{Z}, a d-b c=1,
$$

the set of functions $\chi_{i}$ transforms linearly, and in fact supports a unitary representation of (the double cover of) $P S L(2, \mathbb{Z})$. In particular there exists a unitary matrix $S$ implementing the transformation $\tau \mapsto-1 / \tau$. If $\tilde{q}:=$ $\exp -\frac{2 i \pi}{\tau}$, there exists a unitary $|\mathcal{I}| \times|\mathcal{I}|$ matrix $S$ such that

$$
\chi_{i}(q)=\sum_{j \in \mathcal{I}} S_{i j} \chi_{j}(\tilde{q})
$$

Moreover the matrix $S$ satisfies $S^{T}=S, \quad\left(S_{i j}\right)^{*}=S_{i^{*} j}=S_{i j^{*}}, S^{2}=C=$ the conjugation matrix defined by $C_{i j}=\delta_{i j^{*}}, \quad S^{4}=I{ }^{2}$

For the $c<1$ minimal representations,

$$
\mathcal{I}=\left\{(r, s) \equiv\left(p^{\prime}-r, p-s\right) ; 1 \leq r \leq p^{\prime}-1,1 \leq s \leq p-1\right\}
$$

2 The fact that $S^{2}=C$ rather than $S^{2}=I$ as expected from the transformation $\tau \mapsto-1 / \tau$ signals that we are dealing with a representation of a double covering of the modular group. 
and the $S$ matrix reads

$$
S_{(r, s),\left(r^{\prime} s^{\prime}\right)}=\sqrt{\frac{8}{p p^{\prime}}}(-1)^{(r+s)\left(r^{\prime}+s^{\prime}\right)} \sin \pi r r^{\prime} \frac{p-p^{\prime}}{p^{\prime}} \sin \pi s s^{\prime} \frac{p-p^{\prime}}{p}
$$

For the $\widehat{s l}(2)$ affine algebra, at level $k$, for which $\mathcal{I}=\{1,2, \cdots, k+1\}$, one finds

$$
S_{j j^{\prime}}=\sqrt{\frac{2}{k+2}} \sin \frac{\pi j j^{\prime}}{k+2}, \quad j, j^{\prime} \in \mathcal{I} .
$$

The expression for more general affine algebras may be found in [6]. For non-specialised characters, the transformation reads:

$$
\chi_{i}(q, u)=e^{-i k \pi u^{2} / \tau} \sum_{j \in \mathcal{I}} S_{i j} \chi_{j}(\tilde{q},-u / \tau) .
$$

One notes that the $S$ matrix of the minimal case (1.9) is "almost" the tensor product of two matrices of the form (1.10), at two different levels $k=p-2$ and $k^{\prime}=p^{\prime}-2$. This would be true for $\left|p-p^{\prime}\right|=1$ and if one could omit the identification $(r, s) \equiv\left(p^{\prime}-r, p-s\right)$. This is of course not a coincidence but reflects the "coset construction" of $c<1$ representations of Vir out of the affine algebra $\widehat{s l}(2)$ [7]. We shall encounter below again this fact that minimal cases are "almost" the tensor products of the $\widehat{s l}(2)$ ones.

\section{Fusion Algebra}

Another concept of crucial importance for our discussion is that of fusion algebra. Fusion is an associative and commutative operation among representations of chiral algebras of RCFTs, inherited from the operator product algebra of Quantum Field Theory. It looks similar to the usual tensor product of representations, but contrary to the latter, it is consistent with the finiteness of the set $\mathcal{I}$ and it preserves the central elements (instead of adding them). We shall refer to the literature [8] for a systematic discussion of this concept, and just introduce a notation $\star$ to denote it and distinguish it from the tensor product. It is natural to decompose the fusion of two representations of a chiral algebra on the irreducible representations, thus defining "fusion multiplicities"

$$
\mathcal{V}_{i} \star \mathcal{V}_{j}=\oplus_{k} \mathcal{N}_{i j}^{k} \mathcal{V}_{k}, \quad \mathcal{N}_{i j}{ }^{k} \in \mathbb{N}
$$

There is a remarkable formula, due to Verlinde [9], expressing these multiplicities in terms of the unitary matrix $S$ :

$$
\mathcal{N}_{i j}{ }^{k}=\sum_{\ell \in \mathcal{I}} \frac{S_{i \ell} S_{j \ell}\left(S_{k \ell}\right)^{*}}{S_{1 \ell}}
$$


We will restrict to RCFT for which the Verlinde formula (1.13) produces non-negative integers; this excludes e.g., the fractional level admissible representations of affine algebras, for which there is no such direct relation between the fusion and modular properties.

Note that, regarded as matrices $\mathcal{N}_{i}=\left\{\mathcal{N}_{i j}{ }^{k}\right\}$, the $\mathcal{N}$ form a representation (the regular representation) of the fusion algebra

$$
\mathcal{N}_{i} \mathcal{N}_{j}=\sum_{k \in \mathcal{I}} \mathcal{N}_{i j}{ }^{k} \mathcal{N}_{k}
$$

\section{Chiral Vertex Operators}

The field theoretic description of chiral halves of CFT (or "chiral cft") makes use of "chiral vertex operators" $(\mathrm{CVO}): \phi_{i j}^{k}(z)$ is a $z$-dependent homomorphism from $\mathcal{V}_{i} \star \mathcal{V}_{j}$ to $\mathcal{V}_{k}$ and may be diagrammatically depicted as

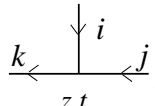

In fact, there are as many as $\mathcal{N}_{i j}^{k}$ such independent intertwiners and the notation $\phi_{i j ; t}^{k}(z)$ and the diagram have to be supplemented by a multiplicity label $t \in\left\{1,2, \cdots, \mathcal{N}_{i j}{ }^{k}\right\}$. CVO may be composed ("fused") as $\phi_{i l}^{m}\left(z_{1}\right) \phi_{j k}^{l}\left(z_{2}\right)$ and there is an invertible matrix $F_{l p}\left[\begin{array}{cc}i & j \\ m & k\end{array}\right]_{t_{1} t_{2}}^{u t}$ expressing that there are two distinct but equivalent ways of intertwining $\mathcal{V}_{i} \star \mathcal{V}_{j} \star \mathcal{V}_{k}$ and $\mathcal{V}_{m}$ :

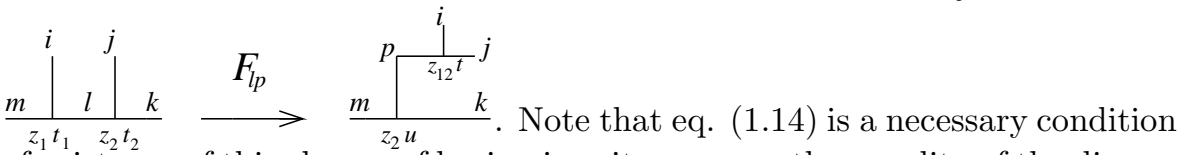
of existence of this change of basis, since it expresses the equality of the dimensions of the two sides: $\sum_{l} \mathcal{N}_{j k}{ }^{l} \mathcal{N}_{i l}{ }^{m}=\sum_{p} \mathcal{N}_{i j}{ }^{p} \mathcal{N}_{p k}{ }^{m}$. The matrix $F$ satisfies a quintic ("pentagon") identity expressing the consistency (the associativity) in the fusion of three CVOs. There is also the operation of braiding and the matrix $B_{l p}\left[\begin{array}{cc}i & j \\ m & k\end{array}\right]( \pm)$ which relates $\phi_{i l}^{m}\left(z_{1}\right) \phi_{j k}^{l}\left(z_{2}\right)$ and $\phi_{j p}^{m}\left(z_{2}\right) \phi_{i k}^{p}\left(z_{1}\right)$, see [10] for a systematic discussion.

Notice finally that the matrix $S$ admits an extension, denoted $S(j)$, such that $S(1)=S$. The matrix $S(p)$ gives the modular transformation of 1-point conformal block $\left\langle\phi_{p i}^{i}\right\rangle_{\tau}$ on the torus. See [10] for the explicit expression of $S(p)$ in the simplest cases.

The data $c$ (or $k$, etc), $\left\{\mathcal{V}_{i}, h_{i}\right\}_{i \in \mathcal{I}}, S(p)_{i j}, \mathcal{N}_{i j}{ }^{k}, F\left[\begin{array}{ll}i & j \\ k & l\end{array}\right]$ and $B\left[\begin{array}{ll}i & j \\ k & l\end{array}\right]( \pm)$ form what may be called the "chiral data" of the RCFT. They are the basic ingredients in the construction of physical theories. The consistency of the latter imposes however that adequate conditions be satisfied by their spectral data and by the structure constants of their Operator Product Algebra (OPA). This is what we shall consider now, examining in turn the cases of the theory "in the bulk", i.e. in the absence of any boundary, and then in the half-plane. 


\subsection{Spectral and OPA Data in the Bulk}

In the plane punctured at the origin, equipped with the coordinate $z$, or equivalently on the cylinder of perimeter $l$ with the coordinate $w$, with the conformal mapping from the latter to the former $z=\exp -2 \pi i w / l$, a given RCFT is described by a Hilbert space $\mathcal{H}_{P}$. This Hilbert space is decomposable into a finite sum of irreducible representations of two copies of the chiral algebra (Vir or else), associated with the holomorphic and anti-holomorphic sectors of the theory:

$$
\mathcal{H}_{P}=\oplus Z_{j \bar{j}} \mathcal{V}_{j} \otimes \overline{\mathcal{V}_{\bar{j}}},
$$

with (non negative integer) multiplicities $Z_{j \bar{j}}$. By the state-field correspondence, (1.15) also describes the spectrum of primary fields of the theory, i.e. of those fields that transform as heighest weight representations of $\mathfrak{A} \otimes \mathfrak{A}$.

A convenient way to encode the information (1.15) is to look at the partition function of the theory on a torus $\mathbb{T}$. Up to a global dilatation, irrelevant here, a torus may be defined by its modular parameter $\tau, \Im m \tau>0$, such that its two periods are 1 and $\tau$. Equivalently, it may be regarded as the quotient of the complex plane by the lattice generated by the two numbers 1 and $\tau$ :

$$
\mathbb{T}=\mathbb{C} /(\mathbb{Z} \oplus \tau \mathbb{Z}),
$$

in the sense that points in the complex plane are identified according to $w \sim$ $w^{\prime}=w+n+m \tau, n, m \in \mathbb{Z}$. There is, however, a redundancy in this description of the torus: the modular parameters $\tau$ and $M \tau$ describe the same torus, for any modular transformation $M \in P S L(2, \mathbb{Z})$. The partition function of the theory on this torus is the trace of the evolution operator $e^{-H}$ on the finite cylinder of period $l=1$, described by $w=\tau v-u \sim w+n, 0 \leq u, v \leq 1$. We have

$$
H=\tau \frac{1}{2 \pi i} \int_{0}^{-1} d u T(w)-\bar{\tau} \frac{1}{2 \pi i} \int_{0}^{-1} d u \bar{T}(\bar{w})
$$

and hence, mapping back to the plane $w \rightarrow z=e^{-2 \pi i w}$ and using the transformation law of the energy-momentum tensor (1.1), we get

$$
Z=\operatorname{tr}_{\mathcal{H}_{P}} e^{2 \pi i\left[\tau\left(L_{0}-\frac{c}{24}\right)-\bar{\tau}\left(\bar{L}_{0}-\frac{c}{24}\right)\right]}
$$

with the trace taking care of the identification of the two ends of the cylinder into a torus. Using (1.15) and the definition (1.3) of characters, this trace may be written as

$$
Z=\sum Z_{j \bar{j}} \chi_{j}(q) \chi_{\bar{j}}(\bar{q}) \quad q=e^{2 \pi i \tau} \quad \bar{q}=e^{-2 \pi i \bar{\tau}} .
$$


Let's stress that in these expressions, $\bar{\tau}$ is the complex conjugate of $\tau$, and $\bar{q}$ that of $q$, and therefore, $Z=\sum Z_{j \bar{j}} \chi_{j}(q)\left(\chi_{\bar{j}}(q)\right)^{*}$ is a sesquilinear form in the characters. It is a natural physical requirement that this partition function be intrinsically attached to the torus, and thus be invariant under modular transformations. Finally one imposes the extra condition $Z_{11}=1$ which expresses the unicity of the identity representation (i.e. of the "vacuum").

One is thus led to the problem of finding all possible sesquilinear forms (1.19) with non negative integer coefficients that are modular invariant, and such that $Z_{11}=1$. As explained in the previous section, the finite set of characters of any RCFT, labelled by $\mathcal{I}$, supports a unitary representation of the modular group. This implies that any diagonal combination of characters $Z=\sum_{i \in \mathcal{I}} \chi_{i}(q) \chi_{i}(\bar{q})$ is modular invariant. In that case, all representations of $\mathfrak{A} \otimes \mathfrak{A}$ appearing in (1.15) are left-right symmetric, and thus all primary fields are spinless: $h_{j}-h_{\bar{j}}=0$. This situation is referred to as the "diagonal case" or "diagonal theory". Other solutions are, however, known to exist.

The problem has been completely solved only in a few cases: for the RCFTs with an affine algebra, the $\widehat{s l}(2)[11]$ and $\widehat{s l}(3)[12]$ theories at arbitrary level, plus a host of cases with constraints on the level, e.g. the general $\widehat{s l}(N)$ for $k=$ $1[13]$; some of the associated coset theories [7] have also been fully classified, including all the minimal $c<1$ theories, $N=2$ "minimal" superconformal theories, etc. A good review on the current state of the art is provided by T. Gannon [14]. For a short account of the cases of $\widehat{s l}(2)$ and $\widehat{s l}(3)$, see [15].

In the case of CFTs with a current algebra, it is in fact better to look at the same problem of modular invariants after replacing in (1.19) all specialized characters by non-specialized ones, v.i.z. $\sum Z_{j \bar{j}} \chi_{j}(q, \mathbf{u})\left(\chi_{\bar{j}}(q, \mathbf{u})\right)^{*}$. Because these non-specialized characters are linearly independent, there is no ambiguity in the determination of the multiplicities $Z_{j} \bar{j}$ from $Z$. This alternative form of the partition function may be seen to result from a modification of the energy-momentum tensor $T(z) \rightarrow T(z)-\frac{2 \pi i}{L}(\mathbf{u}, \mathbf{J}(z))-\frac{k}{2}\left(\frac{2 \pi}{L}\right)^{2}(\mathbf{u}, \mathbf{u})$, see [2].

The matrix $Z_{j \bar{j}}$ of (1.15) gives us the spectrum of primary fields of the theory. We have also to determine the couplings of these fields. This may be done by expressing them in terms of the CVO as

$$
\Phi_{(i, \bar{i})}(z, \bar{z})=\sum_{j, \bar{j}, k, \bar{k}, t, \bar{t}} d_{(i, \bar{i})(j, \bar{j})}^{(k, \bar{k}) t, \bar{t}} \phi_{i, j ; t}^{k}(z) \otimes \phi_{\bar{i}, \bar{j} ; \bar{t}}^{\bar{k}}(\bar{z}) .
$$

Then matrix elements of $\Phi_{(i, \bar{i})}$ between highest weight states are given in terms of those of CVO, which are supposed to be known

$$
\left\langle k, \bar{k}\left|\Phi_{(i, \bar{i})}(1,1)\right| j, \bar{j}\right\rangle=\sum_{t, \bar{t}} d_{(i, \bar{i})(j, \bar{j})}^{(k, \bar{k}) ; t \bar{t}}\left\langle k\left|\phi_{i, j ; t}^{k}(1)\right| j\right\rangle\left\langle\bar{k}\left|\phi_{i, \bar{j} ; \bar{t}}^{\bar{k}}(1)\right| \bar{j}\right\rangle .
$$


For $\widehat{s l}(2)$ related theories, the latter have been explicitly computed in [16,17]. The chiral 3-point blocks can be normalised so that in the diagonal theories the $d$ coefficients are equal to a product of Kronecker delta symbols $\delta_{i \bar{i}} \delta_{j \bar{j}} \delta_{k \bar{k}} \delta_{t \bar{t}}$ with $t=1, \cdots, \mathcal{N}_{i j}{ }^{k}$ implying that $d$ vanishes if $\mathcal{N}_{i j}{ }^{k}=0$ and $\sum_{t, \bar{t}} d_{(i, i)(j, j)}^{(k, k) ; t \bar{t}}=\mathcal{N}_{i j}{ }^{k}$. Thus the expansion coefficients $d_{(i, \bar{i})(j, \bar{j})}^{(k, \bar{t}) ; \bar{t}}$ give in general the relative OPE coefficients of the non-diagonal model with respect to the diagonal model of same central charge. These numbers are constrained by the requirement of locality of the physical correlators, which makes use of the braiding matrices $B( \pm)$. The resulting set of coupled quadratic equations has been fully solved only in the $s l(2)$ cases (see $[16,18]$ and further references therein).

A curious empirical fact was then observed: one may introduce graphs, whose properties are intimately connected with some features of the modular invariants or of the structure constants. In the simplest case of $\widehat{s l}(2)_{k}$ theories, these graphs are the well-known ADE Dynkin diagrams of Coxeter number $h=k+2$, and the set of labels $j$ of the diagonal terms appearing in $Z$ in (1.19) is precisely the set of so-called exponents labelling the eigenvalues $2 \cos \pi j / h$ of the adjacency matrix of the diagram (see Table 1). Moreover let's introduce the corresponding eigenvectors $\psi_{a}^{j}$ of the adjacency matrix, ( $a$ being a vertex of the diagram $G$ ) and define the structure constants of the so-called Pasquier algebra [19]

$$
M_{i j}{ }^{k}=\sum_{a \in G} \frac{\psi_{a}^{i} \psi_{a}^{j}\left(\psi_{a}^{k}\right)^{*}}{\psi_{a}^{1}}
$$

Then the structure constants $d_{(i, i),(j, j)}{ }^{k, k)}$ of spinless fields in the theory may be shown to be equal to those of the Pasquier algebra $d_{(i, i),(j, j}{ }^{k, k)}=M_{i j}{ }^{k}[18]$. Thus the ADE graph encodes some non trivial information about a subsector of the theory, namely that of spinless fields, their spectrum and OPE.

These empirical facts are known to extend to more complicated theories. In general, we expect that among the pairs $(j, \bar{j})$ appearing in $Z$, a special role will be played by the diagonal subset

$$
\mathcal{E}=\left\{(j, \alpha) \mid \alpha=1, \cdots Z_{j j}\right\}
$$

the elements of which, the "exponents" of the theory, are thus counted with the multiplicity $Z_{j j}$. (In what follows, we shall most of the time omit the multiplicity index $\alpha$ ). We assume that $\mathcal{E}$ is stable under conjugation: $j$ and $j^{*}$ occur with the same multiplicity. Then the relevant graphs $G_{i}$ are labelled by the set $\mathcal{I}$, they have a common set of vertices and their spectrum is described 
by the set $\mathcal{E}$ in the sense that their eigenvalues are of the form $S_{i j} / S_{1 j}$ and of multiplicity $Z_{j j}$.

The origin and interpretation of these empirical facts had remained elusive until recently. It is one of the virtues of BCFT to have cast a new light on these facts and to have offered a new framework in which they appear more natural and systematic.

Table 1: ADE graphs, their Coxeter number and their exponents

$$
h \quad \text { exponents }
$$
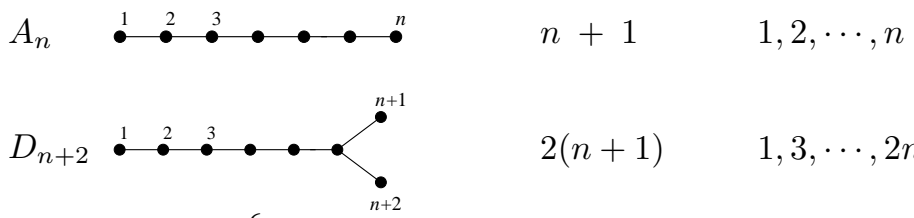

$$
2(n+1) \quad 1,3, \cdots, 2 n+1, n+1
$$

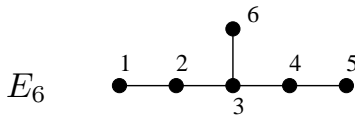

12

$1,4,5,7,8,11$

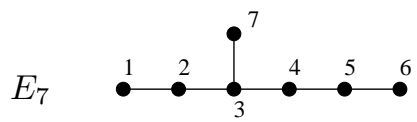

18

$1,5,7,9,11,13,17$

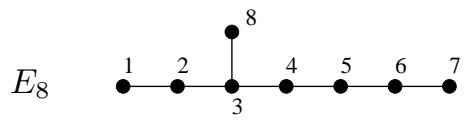

\section{Boundary Conformal Field Theory}

\subsection{Spectral Data in the Upper Half Plane}

We now turn to the study of RCFT in a half-plane. There are several physical reasons to look at this problem, as mentioned in the Introduction. Here we shall only look at the new information and perspective that this situation yields on the general structure of RCFT.

In a half-plane, the admissible diffeomorphisms must respect the boundary, taken as the real axis: thus only real analytic changes of coordinates, satisfying $\epsilon(z)=\bar{\epsilon}(\bar{z})$ for $z=\bar{z}$ real, are allowed. The energy momentum itself has this property:

$$
T(z)=\left.\bar{T}(\bar{z})\right|_{\text {real axis }},
$$


which expresses simply the absence of momentum flow across the boundary and which enables one to extend the definition of $T$ to the lower half-plane by $T(z):=\bar{T}(z)$ for $\Im m z<0$. There is thus only one copy of the Virasoro algebra $L_{n}=\bar{L}_{n}$. This continuity equation (2.1) on $T$ extends to more general chiral algebras and their generators, at the price however of some complication. In general, the continuity equation on generators of the chiral algebra involves some automorphism of that algebra:

$$
W(z)=\left.\Omega \bar{W}(\bar{z})\right|_{\text {real axis }}
$$

(see [2] and further references therein).

The half-plane, punctured at the origin, (which introduces a distinction between the two halves of the real axis), may also be conformally mapped on an infinite horizontal strip of width $L$ by $w=\frac{L}{\pi} \log z$. Boundary conditions, loosely specified at this stage by labels $a$ and $b$, are assigned to fields on the two boundaries $z$ real $>0,<0$ or $\Im m w=0, L$. For given boundary conditions on the generators of the algebra and on the other fields of the theory, i.e. for given automorphisms $\Omega$ and given $a, b$, we may again use a description of the system by a Hilbert space of states $\mathcal{H}_{b a}$ (we drop the dependence on $\Omega$ for simplicity). This space decomposes on representations of Vir or $\mathfrak{A}$ according to

$$
\mathcal{H}_{b a}=\oplus n_{i b}{ }^{a} \mathcal{V}_{i}
$$

with a new set of multiplicities $n_{i b}{ }^{a} \in \mathbb{N}$. The natural Hamiltonian on the strip is the translation operator in $\Re e w$, hence, mapped back in the half-plane

$$
H_{b \mid a}=\frac{\pi}{L}\left(L_{0}-\frac{c}{24}\right) .
$$

To recapitulate, in order to fully specify the operator content of the theory in various configurations, we need not only determine the multiplicities "in the bulk" $Z_{j \bar{j}}$ of (1.15), but also the possible boundary conditions $a, b$ on a half-plane and the associated multiplicities $n_{i b}{ }^{a}$. This will be our task in the following, and as we shall see, a surprising fact is that the latter have some bearing on the former.

\subsection{Boundary states}

In the same way that we found useful to chop a finite segment of the infinite cylinder and identify its ends to make a torus, it is suggested to consider a finite segment of the strip - or a semi-annular domain in the half-plane--and identify 

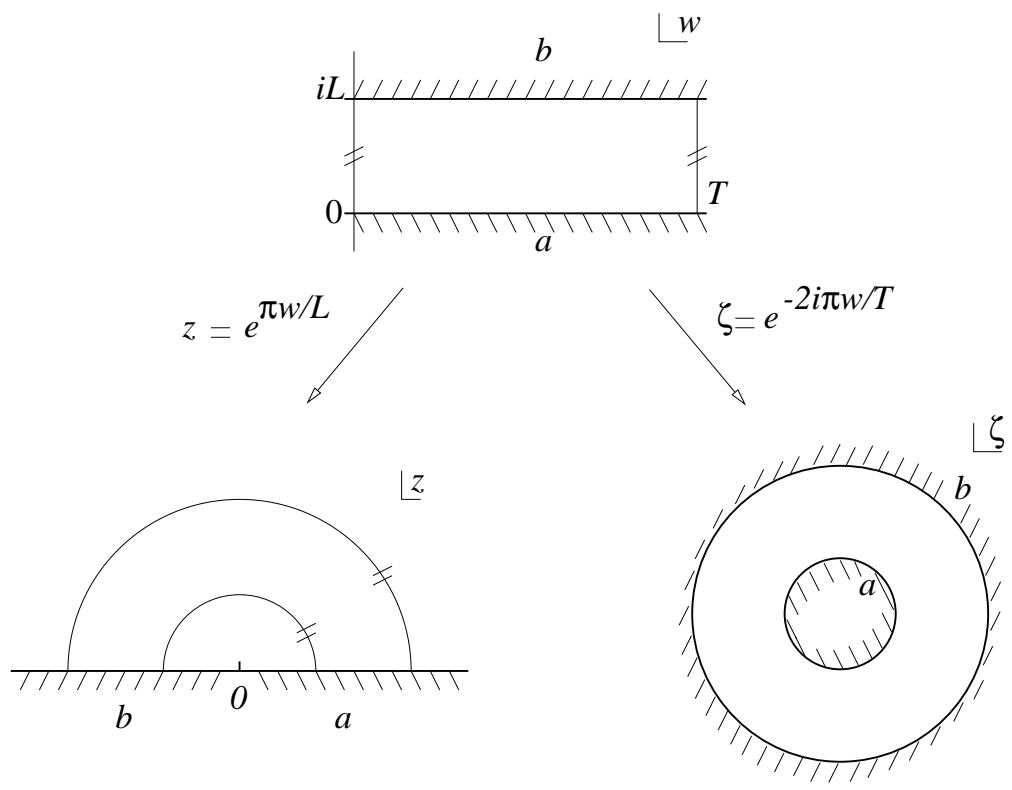

Fig. 1: The same domain seen in different coordinates: a semi-circular annulus, with the two half-circles identified, a rectangular domain with two opposite sides identified, and a circular annulus.

its edges, thus making a cylinder. This cylinder can be mapped back into an annular domain in the plane, with open boundaries. More explicitly, consider the segment $0 \leq \Re e w \leq T$ of the strip -i.e. the semi-annular domain in the upper half-plane comprised between the semi-circles of radii 1 and $e^{\pi T / L}$, the latter being identified. It may be conformally mapped into an annulus in the complex plane by $\zeta=\exp (-2 i \pi w / T)$, of radii 1 and $e^{2 \pi L / T}$, see Fig. 1. By working out the effect of this change of coordinates on the energy-momentum $T$, using (1.1), one finds that (2.1) implies

$$
\zeta^{2} T(\zeta)=\bar{\zeta}^{2} \bar{T}(\bar{\zeta}) \quad \text { for }|\zeta|=1, e^{2 \pi \frac{L}{T}}
$$

After radial quantization, this translates into a condition on boundary states $|a\rangle,|b\rangle \in \mathcal{H}_{P}$ which describe the system on these two boundaries.

$$
\left(L_{n}-\bar{L}_{-n}\right)|a\rangle=0
$$

(and likewise for $|b\rangle$ ). 
Exercise : assuming that $W$ transforms as a primary field of conformal weight $\left(h_{W}, 0\right)$, find the corresponding condition on $W(\zeta)$. Then show that the analogue of (2.6) reads $\left(W_{n}-(-1)^{h_{W}} \Omega\left(\bar{W}_{-n}\right)\right)|a\rangle=0$.

We shall now look for a basis of states, solutions of this linear system of boundary conditions. One may seek solutions of these equations in each $\mathcal{V}_{j} \otimes \mathcal{V}_{\bar{j}} \subset \mathcal{H}_{P}$, since these spaces are invariant under the action of the two copies of Vir or of the chiral algebra $\mathfrak{A}$. Consider only for simplicity the case of the Virasoro generators.

Lemma There is an independent "Ishibashi state" $|j\rangle\rangle$, solution of (2.6), for each $j=\bar{j}$, i.e. $j \in \mathcal{E}$, the set of exponents.

Proof (G. Watts)[20]: Use the identification between states $|a\rangle \in \mathcal{V}_{j} \otimes \mathcal{V}_{\bar{j}}$ and operators $X_{a} \in \operatorname{Hom}\left(\mathcal{V}_{\bar{j}}, \mathcal{V}_{j}\right)$, namely $|a\rangle=\sum_{n, \bar{n}} a_{n, \bar{n}}|j, n\rangle \otimes|\bar{j}, \bar{n}\rangle \leftrightarrow X_{a}=$ $\sum_{n, \bar{n}} a_{n, \bar{n}}|j, n\rangle\langle\bar{j}, \bar{n}|$. Here we make use of the scalar product in $\mathcal{V}_{\bar{j}}$ for which $\bar{L}_{-n}=\bar{L}_{n}^{\dagger}$, hence (2.6) means that $L_{n} X_{a}=X_{a} L_{n}$, i.e. $X_{a}$ intertwines the action of Vir in the two irreducible representations $\mathcal{V}_{j}$ and $\mathcal{V}_{\bar{j}}$. By Schur's lemma, this implies that they are equivalent, $\mathcal{V}_{j} \sim \mathcal{V}_{\bar{j}}$, i.e. that their labels coincide $j=\bar{j}$ and that $X_{a}$ is proportional to $P_{j}$, the projector in $\mathcal{V}_{j}$. We shall denote $|j\rangle\rangle$ the corresponding state, solution to (2.6).

Since "exponents" $j \in \mathcal{E}$ may have some multiplicity, an extra label should be appended to our notation $|j\rangle\rangle$. We omit it for the sake of simplicity. The previous considerations extend with only notational complications to more general chiral algebras and their possible gluing automorphisms $\Omega$. See [2] for more details and more references on these points. Also, see [21] for an alternative discussion of Ishibashi states.

The normalization of this "Ishibashi state" requires some care. One first notices that, for $\tilde{q}$ a real number between 0 and 1 ,

$$
\left\langle\left\langle j^{\prime}\left|\tilde{q}^{\frac{1}{2}\left(L_{0}+\bar{L}_{0}-\frac{c}{12}\right)}\right| j\right\rangle\right\rangle=\delta_{j j^{\prime}} \chi_{j}(\tilde{q})
$$

up to a constant that we choose equal to 1 . It would seem natural to then define the norm of these states by the limit $\tilde{q} \rightarrow 1$ of (2.7). This limit diverges, however, and the adequate definition is rather

$$
\left\langle\left\langle j \| j^{\prime}\right\rangle\right\rangle=\delta_{j j^{\prime}} S_{1 j}
$$

This comes about in the following way: a natural regularization of the above limit is:

$$
\left\langle\left\langle j \| j^{\prime}\right\rangle\right\rangle=\lim _{\tilde{q} \rightarrow 1} q^{c / 24}\left\langle\left\langle j^{\prime}\left|\tilde{q}^{\frac{1}{2}\left(L_{0}+\bar{L}_{0}-\frac{c}{12}\right)}\right| j\right\rangle\right\rangle
$$


where $q$ is the modular transform of $\tilde{q}=e^{-2 \pi i / \tau}, q=e^{2 \pi i \tau}$. In a ("unitary") theory in which the identity representation (denoted 1 ) is the one with the smallest conformal weight, show that in the limit $q \rightarrow 0$, the r.h.s. of (2.9) reduces to (2.8). In non unitary theories, this limiting procedure fails, but we keep (2.8) as a definition of the new norm.

At the term of this study, we have found a basis of solutions to the constraint (2.6) on boundary states, and it is thus legitimate to expand the two states attached to the two boundaries of our domain as

$$
\left.|a\rangle=\sum_{j \in \mathcal{E}} \frac{\psi_{a}^{j}}{\sqrt{S_{1 j}}}|j\rangle\right\rangle
$$

with coefficients denoted $\psi_{a}^{j}$, and likewise for $|b\rangle$. We define an involution $a \rightarrow a^{*}$ on the boundary states by $\psi_{a^{*}}^{j}=\psi_{a}^{j^{*}}=\left(\psi_{a}^{j}\right)^{*}$, (recall that $j \rightarrow j^{*}$ is an involution in $\mathcal{E})$. One may show [22] that it is natural to write for the conjugate state

$$
\langle b|=\sum_{j \in \mathcal{E}}\left\langle\langle j| \frac{\psi_{b^{*}}^{j}}{\sqrt{S_{1 j}}} .\right.
$$

As a consequence

$$
\langle b \| a\rangle=\sum_{j \in \mathcal{E}} \frac{\psi_{a}^{j}\left(\psi_{b}^{j}\right)^{*}}{S_{1 j}}\langle\langle j \| j\rangle\rangle=\sum_{j \in \mathcal{E}} \psi_{a}^{j}\left(\psi_{b}^{j}\right)^{*}
$$

so that the orthonormality of the boundary states is equivalent to that of the $\psi$ 's.

\subsection{Cardy equation}

Let us return to the annulus $1 \leq|\zeta| \leq e^{2 \pi L / T}$ considered in last subsection, or equivalently to the cylinder of length $L$ and perimeter $T$, with boundary conditions (b.c.) $a$ and $b$ on its two ends. Following Cardy [23], we shall compute its partition function $Z_{b \mid a}$ in two different ways. If we regard it as resulting from the evolution between the boundary states $|a\rangle$ and $\langle b|$, with $\tilde{q}^{\frac{1}{2}}=e^{-2 \pi L / T}$, we find

$$
\begin{aligned}
Z_{b \mid a} & =\langle b|\left(\tilde{q}^{\frac{1}{2}\left(L_{0}+\bar{L}_{0}-\frac{c}{12}\right)}|a\rangle=\sum_{j, j^{\prime} \in \mathcal{E}} \frac{\left(\psi_{b}^{j}\right)^{*} \psi_{a}^{j^{\prime}}}{S_{1 j}}\left\langle\left\langle j\left|\tilde{q}^{\frac{1}{2}\left(L_{0}+\bar{L}_{0}-\frac{c}{12}\right)}\right| j^{\prime}\right\rangle\right\rangle\right. \\
& =\sum_{j \in \mathcal{E}} \psi_{a}^{j}\left(\psi_{b}^{j}\right) * \frac{\chi_{j}(\tilde{q})}{S_{1 j}} .
\end{aligned}
$$




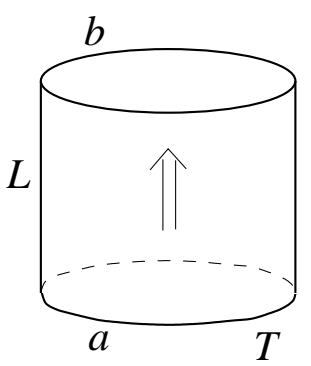

(a)

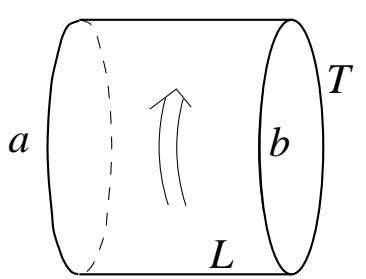

(b)

Fig. 2: Two alternative computations of the partition function $Z_{b \mid a}$ : (a) on the cylinder, between the boundary states $|a\rangle$ and $\langle b|,(\mathrm{b})$ as a periodic time evolution on the strip, with boundary conditions $a$ and $b$.

On the other hand, if we regard it as resulting from the periodic "time" evolution on the strip with b.c. $a$ and $b$, using the decomposition (2.3) of the Hilbert space $\mathcal{H}_{b a}$, and with $q=e^{-\pi T / L}$

$$
Z_{b \mid a}(q)=\sum_{i \in \mathcal{I}} n_{i b}^{a} \chi_{i}(q)
$$

See Fig. 2. Note that string theorists would refer to these two situations as (a): the tree approximation of the propagation of a closed string; (b) the oneloop evolution of an open string. Performing a modular transformation on the characters $\chi_{j}(\tilde{q})=\sum_{i} S_{j i^{*}} \chi_{i}(q)$ in (2.13), and identifying the coefficients of $\chi_{i}$ yields

$$
n_{i a}{ }^{b}=\sum_{j \in \mathcal{E}} \frac{S_{i j}}{S_{1 j}} \psi_{a}^{j}\left(\psi_{b}^{j}\right)^{*},
$$

a fundamental equation for our discussion that we refer to as Cardy equation [23]. In deriving this form of Cardy equation, we have made use of a symmetry property of $n_{i a}{ }^{b}$

$$
n_{i a}{ }^{b}=n_{i^{*} b}{ }^{a}
$$

which follows from the symmetries of $S$.

(Comment: this identification of coefficients of specialized characters is in general not justified, as the $\chi_{i}(q)$ are not linearly independent. As in sect. 1, it is better to generalize the previous discussion, in a way which introduces non-specialized -and linearly independentcharacters. This has been done in [2] for the case of CFTs with a current algebra. Unfortunately, little is known about non-specialized characters for other chiral algebras.) 
Let us stress that in (2.15), the summation runs over $j \in \mathcal{E}$, i.e. this equation incorporates some information on the spectrum of the theory "in the bulk", i.e. on the modular invariant partition function (1.19).

Cardy equation (2.15) is a non linear constraint relating a priori unknown complex coefficients $\psi^{j}$ to unknown integer multiplicities $n_{i a}{ }^{b}$. We need additional assumptions to exploit it.

We shall thus assume that

- we have found an orthonormal set of boundary states $|a\rangle$, i.e. satisfying

$$
\left(n_{1}\right)_{a}{ }^{b}=\sum_{j \in \mathcal{E}} \psi_{a}^{j}\left(\psi_{b}^{j}\right)^{*}=\delta_{a b}
$$

- we have been able to construct a complete set of such boundary states $|a\rangle$

$$
\sum_{a} \psi_{a}^{j}\left(\psi_{a}^{j^{\prime}}\right)^{*}=\delta_{j j^{\prime}}
$$

These assumptions imply that

$$
\# \text { boundary states }=\# \text { independent Ishibashi states }=|\mathcal{E}| \text {. }
$$

None of these assumptions is innocent. In particular, it is in general not consistent to assume that all boundary states are mutually orthogonal; for example it has been shown recently that the renormalisation group flow may bring a theory to a boundary state where orthogonality is violated $[24,25,5]$. Such a state $A$ is a linear superposition with non-negative integer coefficients of boundary states satisfying (2.17), see the exercise below. As for the assumption of completeness, it is not obviously natural and would also deserve a critical discussion; relaxing it would force us to reconsider the forthcoming discussion.

Exercise: Suppose we have found a complete and orthonormal solution $\{\psi\}$ to (2.15) and consider a boundary state $\left.|A\rangle=\sum_{j \in \operatorname{Exp}} \Psi_{A}^{j}\left(S_{1 j}\right)^{-1 / 2}|j\rangle\right\rangle$ which also satisfies (2.15) with itself and with all the orthonormal states $|a\rangle: \sum_{j \in \operatorname{Exp}} \Psi_{A}^{j} \psi_{a}^{j *} S_{i j} / S_{1 j}=N_{i A}{ }^{a} \in \mathbb{N}$. Prove that this implies that

$$
\Psi_{A}^{j}=\sum_{a} N_{1 A^{a}} \psi_{a}^{j},
$$

and that conversely, the integrality of $N_{i A}{ }^{a}$ and of $N_{i A}{ }^{A}$ follows from (2.19), with $N_{1 A}{ }^{a} \in$ $\mathbb{N}$.

\subsection{Representations of the fusion algebra and graphs}

Return to Cardy equation (2.15) supplemented by the above assumptions (2.17)-(2.18) and observe that it gives a decomposition of the matrices $n_{i}$, 
defined by $\left(n_{i}\right)_{a}{ }^{b}=n_{i a}{ }^{b}$, into their orthonormal eigenvectors $\psi$ and their eigenvalues $S_{i j} / S_{1 j}$. Observe also that as a consequence of Verlinde formula (1.13), these eigenvalues form a one-dimensional representation of the fusion algebra

$$
\frac{S_{i \ell}}{S_{1 \ell}} \frac{S_{j \ell}}{S_{1 \ell}}=\sum_{k \in \mathcal{I}} \mathcal{N}_{i j}{ }^{k} \frac{S_{k \ell}}{S_{1 \ell}}, \quad \forall i, j, \ell \in \mathcal{I}
$$

Hence the matrices $n_{i}$ also form a representation of the fusion algebra (1.14)

$$
n_{i} n_{j}=\sum_{k \in \mathcal{I}} \mathcal{N}_{i j}^{k} n_{k}
$$

and they thus commute. Moreover, as we have seen above, they satisfy $n_{1}=I$, $n_{i}^{T}=n_{i^{*}}$.

Conversely, consider any $\mathbb{N}$-valued matrix representation of the Verlinde fusion algebra $n_{i}$, such that $n_{i}^{T}=n_{i^{*}}$. Since the algebra is commutative, $\left[n_{i}, n_{i}^{T}\right]=\left[n_{i}, n_{i^{*}}\right]=0$, the $n_{i}$ commute with their transpose, (normal matrices), hence they are diagonalizable in a common orthonormal basis. Their eigenvalues are known to be of the form $S_{i j} / S_{1 j}$. They may thus be written as in (2.15). Thus any such $\mathbb{N}$-valued matrix representation of the Verlinde fusion algebra gives a (complete orthonormal) solution to Cardy's equation.

\section{Conclusion:}

$\mathbb{N}$-valued matrix representation of the fusion algebra, $n_{i}^{T}=n_{i^{*}}$ $\Longleftrightarrow$ Complete, orthonormal solution of Cardy equation

Moreover, since $\mathbb{N}$-valued matrices are naturally interpreted as graph adjacency matrices, graphs appear naturally and their spectral properties are those anticipated in the last lines of sect. 1.

The relevance of the fusion algebra in the solution of Cardy equation had been pointed out by Cardy himself for diagonal theories [23] and foreseen in general in [26] with no good justification; the importance of the assumption of completeness of boundary conditions was first stressed by Pradisi et al [27].

\subsection{The case of $\widehat{s l}(2)$ WZW theories}

Problem: Classify all $\mathbb{N}$-valued matrix reps of $\widehat{s \ell}(2)_{k}$ fusion algebra with $k$ fixed. 
The algebra is generated recursively by $n_{2}$

$$
\begin{gathered}
n_{1}=I, \quad n_{2} n_{i}=n_{i+1}+n_{i-1}, \quad i=2, \ldots, k \\
\quad S \text { real } \Rightarrow n_{i}=n_{i}^{T} .
\end{gathered}
$$

Even though $\psi^{j}$ and $\mathcal{E}$ are a priori unknown, we know from (1.10) that $n_{2}$ has eigenvalues of the form

$$
\gamma_{j}=\frac{S_{2 j}}{S_{1 j}}=2 \cos \frac{\pi j}{k+2}, \quad j \in \mathcal{E} .
$$

We shall discard the case where the matrix $n_{2}$ is "reducible", i.e. may be written as a direct sum of two matrices $n_{2}=n_{2}^{(1)} \oplus n_{2}^{(2)}$, because then all the other matrices $n_{i}$ have the same property and this corresponds to decoupled boundary conditions. It turns out that irreducible $\mathbb{N}$-valued matrices $G$ with spectrum $|\gamma|<2$ have been classified [28]. They are the adjacency matrices either of the $A-D-E$ Dynkin diagrams or of the "tadpoles" $T_{n}=A_{2 n} / \mathbb{Z}_{2}$. Thus as a consequence of equation (2.15) alone, for a $\widehat{s l}(2)$ theory at level $k$, the possible boundary conditions are in one-to-one correspondence with the vertices of one of these diagrams $G$, with Coxeter number $h=k+2$. If we remember, however, that the set $\mathcal{E}$ must appear in one of the modular invariant torus partition functions, the case $G=T_{n}$ has to be discarded, and we are left with $A D E$. (Up to this last step, this looks like the simplest route leading to the $A D E$ classification of $\widehat{s l}(2)$ theories.) We thus conclude that for each $\widehat{s l}(2)$ theory classified by a Dynkin diagram $G$ of $A D E$ type

$$
\mathcal{E}=\operatorname{Exp}(G), \quad \operatorname{dim}\left(n_{i}\right)=|\mathcal{E}|=|G|
$$

complete orthonormal b. c. $=a, b, \cdots$ : vertices of $G$

$$
\begin{aligned}
n_{2} & =\text { adjacency matrix of } G \\
n_{i} & =\text { " } i \text {-th fused adjacency matrix" of } G \\
\psi^{j} & =\text { eigenvector of } n_{2} \text { with eigenvalue } \gamma_{j} .
\end{aligned}
$$

One checks indeed that the matrices $n_{i}$, given by equation (2.15), together with (1.10), have only non negative integer elements. See $[2,15]$ for a review of their remarkable properties and of their ubiquitous rôle in a variety of problems.

\subsection{The case of $c<1$ minimal models}

As recalled above, this case is closely related to the $\widehat{s l}(2)$ models that we just discussed. If $c=1-\frac{6\left(p-p^{\prime}\right)^{2}}{p p^{\prime}}$, as in sect 1.1 , the classification of modular 
invariants is done by pairs of Dynkin diagrams $\left(A_{p^{\prime}-1}, G\right)$, with $p$ equal to the Coxeter number of $G$. Our problem is then to classify all $\mathbb{N}$-valued matrix representations of the corresponding fusion algebra.

Theorem: [2] The only complete orthonormal solution to Cardy's equation are labelled by pairs $(r, a)$ of nodes of the $A_{p^{\prime}-1}$ and of the $G$ graphs, with the identification

$$
(r, a) \equiv\left(p^{\prime}-r, \gamma(a)\right)
$$

where $\gamma$ is the following automorphism of the $G$ Dynkin diagram: the natural $\mathbb{Z}_{2}$ symmetry for the $A, D_{\text {odd }}$ and $E_{6}$ cases, the identity for the others.

The solutions are given explicitly as

$$
n_{r s}=N_{r} \otimes n_{s}^{(G)}+N_{p^{\prime}-r} \otimes n_{p-s}^{(G)}
$$

or

$$
n_{r s ;\left(r_{1}, a\right)}^{\left(r_{2}, b\right)}=N_{r r_{1}}^{r_{2}} n_{s a}^{(G) b}+N_{p^{\prime}-r r_{1}}^{r_{2}} n_{p-s a}^{(G)}{ }^{b},
$$

with $1 \leq r, r_{1}, r_{2} \leq p^{\prime}-1,1 \leq s \leq p-1$, and $a, b$ are running over the nodes of the Dynkin diagram $G ; N_{r}$ are the fusion matrices of $\widehat{s l}(2)_{p^{\prime}-2}, n_{s}^{(G)}$ are the representation matrices of the fusion algebra of $\widehat{s l}(2)_{p-2}$ associated with the graph $G$ and introduced in sect 2.5 .

Hint: Look at the generators $n_{21}$ and $n_{12}$ of fusion algebra. Both have eigenvalues $|\gamma|<2$, but now they are no longer irreducible matrices as in sect. 2.5. To show that (2.26) is indeed the only solution requires a detailed discussion, see [2].

Example: The 3-state Potts model. This is the $p^{\prime}=5, p=6(c=4 / 5)$ minimal model, classified by the pair $\left(A_{4}, D_{4}\right)$. According to the previous discussion, there are 8 distinct boundary conditions labelled by

$$
(r, a) \in\left(A_{4}, D_{4}\right) / \mathbb{Z}_{2} \quad \text { e.g. } r=1,2, a \in D_{4}
$$

This was first pointed out in [29]: the three boundary conditions $(1, a)$ where $a=1,3,4$ is one of the three end-points of the $D_{4}$ diagram are fixed b.c., corresponding to fixing the value of the Potts "spin" to one of its three values, while in the three b.c. $(2, a)$, the boundary spin may take either value different from $a$; the b.c. $(1,2)$, where 2 denotes the middle point of the diagram, is the free boundary condition: the boundary spin may take an arbitrary value; finally the b.c. $(2,2)$ is more delicate to describe [29]. The explicit expressions of the different partition functions of type $Z_{(1,1) \mid(r, a)}$ read

$$
\begin{aligned}
& Z_{(1,1) \mid(1,1)}=\chi_{(1,1)}+\chi_{(1,5)} \\
& Z_{(1,1) \mid(1,2)}=\chi_{(4,2)}+\chi_{(4,4)}
\end{aligned}
$$




$$
\begin{aligned}
& Z_{(1,1) \mid(1, a)}=\chi_{(1,3)} \quad \text { if } a=3,4 \\
& Z_{(1,1) \mid(2,1)}=\chi_{(3,1)}+\chi_{(3,5)} \\
& Z_{(1,1) \mid(2,2)}=\chi_{(2,2)}+\chi_{(2,4)} \\
& Z_{(1,1) \mid(2, a)}=\chi_{(3,3)} \quad \text { if } a=3,4 .
\end{aligned}
$$

The others are linear combinations with non-negative integer coefficients of the latter. See also $[30,31]$ for a lattice realization with integrable boundary weights.

\subsection{Other cases}

It should be clear that the situation that we have described in detail for $s l(2)$ extends to all RCFTs. The matrices $n_{i}$, solutions to Cardy equation, are the adjacency matrices of graphs. In the case of $\widehat{s l}(N)$, it is sufficient to supply the $(N-1)$ fundamental matrices $n_{\square}, p=1, \cdots, N-1$, indexed by the Young

$$
:: \vdots p
$$

tableaux of the fundamental representations of $s l(N)$, to determine all of them. The fact that all $n_{i}$ have non negative integer elements is then non trivial. By Cardy equation again, they satisfy a very restrictive spectral property: their eigenvalues must be of the form $S_{i j} / S_{1 j}$, when $j$ runs over the set $\mathcal{E}$, i.e. the diagonal part of the modular invariant.

The program of classifying these graphs/boundary conditions has been completed only in a few cases: $\widehat{s l}(2)$ as discussed above, $\widehat{s l}(3)$ through a combination of some empirical search of relevant graphs [32], of Gannon's classification of the modular invariants [12], and of the recent work of Ocneanu [33], see $[2,15]$ for a discussion; $\widehat{s l}(N)_{1}[2]$, where the results match those obtained in the study of modular invariants [13]: the graphs turn out to be star polygons.

\section{Boundary Operator Algebra}

According to Cardy [23], changes of boundary conditions can be interpreted as due to the insertion of fields ${ }^{b} \Psi_{j, \beta}^{a}(x)$ living on the boundary, $\Im m z=0$, $x=\Re e z$ of the upper half-plane $z \in \mathbb{H}$. We know the spectrum of these fields from the previous discussion: for a given pair $a, b$ of boundary conditions and a label $j \in \mathcal{I}$, there are $n_{j a}{ }^{b}$ independent such fields, which are thus labelled by a multiplicity label $\alpha=1, \cdots, n_{j a}{ }^{b}$. Pictorially, we may again use a CVOlike representation for ${ }^{b} \Psi_{j, \alpha}^{a}(x)=\frac{b^{j} \downarrow_{\alpha} a}{a}$. It is a natural -and physically important- question to determine the correlation functions of these new fields in the possible presence of the "usual" fields "in the bulk". Two quantities of particular importance are 
i) the fusion matrix ${ }^{(1)} F$ of boundary operators, which plays for the boundary fields the same rôle as the matrix $F$ for the CVO (sect. 1.1),

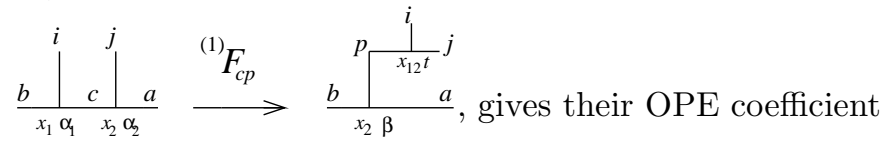

$$
{ }^{b} \Psi_{i, \alpha_{1}}^{c}\left(x_{1}\right){ }^{c} \Psi_{j, \alpha_{2}}^{a}\left(x_{2}\right)=\sum_{p, \beta, t}{ }^{(1)} F_{c p}\left[\begin{array}{cc}
i & j \\
b & a
\end{array}\right]_{\alpha_{1} \alpha_{2}}^{\beta}\left\langle p, 0\left|\phi_{i j ; t}^{p}\left(x_{12}\right)\right| j, 0\right\rangle{ }^{b} \Psi_{p, \beta}^{a}\left(x_{2}\right)+\ldots
$$

in which the multiplicity labels run over $\alpha_{1}=1, \cdots, n_{i c}{ }^{b}, \alpha_{2}=1, \cdots, n_{j a}{ }^{c}$, $\beta=1, \cdots, n_{p a}{ }^{b}, t=1, \cdots, \mathcal{N}_{i j}{ }^{p}$; note that eq. (2.21) is again a necessary condition for the matrix ${ }^{(1)} F$ to be invertible;

ii) the bulk-boundary "reflection" coefficients $R_{a, \alpha}^{\left(i, \bar{i}^{*}, t\right)}(p)$ (denoted ${ }^{a, \alpha} B_{(i, \bar{i})}^{p, t}$ in [2]) enable one to expand bulk fields $\Phi$ in terms of $\Psi$, close to the boundary, i.e. for small $y=\Im m z$

$$
\Phi_{(i, \bar{i})}(z, \bar{z})=\sum_{a, \alpha, p \in \mathcal{I}, t} R_{a, \alpha}^{\left(i, \bar{i}^{*}, t\right)}(p)\left\langle p, 0\left|\phi_{i \bar{i} ; t}^{p}(2 i y)\right| j, 0\right\rangle^{a} \Psi_{p, \alpha}^{a}(x)+\ldots
$$

Here $\alpha=1, \cdots, n_{p a}{ }^{a}, t=1, \cdots, \mathcal{N}_{i \bar{i}} p^{*}$. Given these data (and for a chosen normalisation of the $\Psi$ ), we may in principle compute all correlation functions of $\Psi$ and $\Phi$ [34].

We shall not dwell here on the determination of the fusion matrices ${ }^{(1)} F$ and of the bulk-boundary coefficients. They have been the object of much activity lately, in particular on their connection with chiral data and on the algebraic relations that they satisfy. Their explicit calculation for the $A$ or $D$ type minimal models has been completed by Runkel $[35,36]$. Let us just mention two results:

In the diagonal theories, the matrices ${ }^{(1)} F$ coincide with the standard fusion matrices $F$ : this observation first made in [35] for the $s l(2)$ case through a tedious computation is in accordance with the fact that in that case, the indices $a, b, \cdots$ are of the same nature as $i, j, \cdots$, i.e. also belong to the set $\mathcal{I}$; this result was generally established in [2] rewriting the boundary field Lewellen sewing equation as a pentagon relation, see Fig. 3 below. Also, the reflection coefficients $R(p)$ are expressible in terms of the matrix $S(p)$ introduced at the end of sect. 1.1, a quite remarkable convergence between seemingly very different objects.

In general, the bulk-boundary coefficient pertaining to the identity, i.e. $R_{a, 1}^{\left(i, i^{*}, 1\right)}(1)$ is proportional to $\psi_{a}{ }^{i} / \psi_{a}{ }^{1}$. It thus satisfies, up to a normalisation, 
the Pasquier algebra (1.22),

$$
\frac{\psi_{a}{ }^{i}}{\psi_{a}{ }^{1}} \frac{\psi_{a}^{j}}{\psi_{a}{ }^{1}}=\sum_{k \in \mathcal{I}} M_{i j}{ }^{k} \frac{\psi_{a}{ }^{k}}{\psi_{a}{ }^{1}} .
$$

This expresses the OPA of bulk fields of type $\Phi_{(i, i)}$ near the boundary $(a)$ $[27,22,21,2]$, and must be compared with the empirical observation [18] mentioned in sect. 1.2 on the rôle of the Pasquier algebra in the OPA of spinless fields, now derived in BCFT.

The determination of the ${ }^{(1)} F$ matrix, whose entries are called "cells" by Ocneanu [37], turns out to be an essential step, not only in the study of boundary effects, but also in uncovering hidden algebraic aspects of the theory (sect. 5) and in the study of associated lattice models (sect. 6). For a given set of matrices $\left\{n_{i}\right\}$-a given "graph" - this set of cells satisfies various non linear relations: orthonormality expressing the fact that it plays the rôle of a change of basis (" $3 j$-symbols") in tensors products in a certain space, a mixed pentagon identity written symbolically as $F^{(1)} F^{(1)} F={ }^{(1)} F^{(1)} F$, expressing the consistency (associativity) in the fusion of several boundary fields, (see Fig. 3), and other identities. See $[33,38,39]$ for more details and references.

It is thus a non trivial task to determine ${ }^{(1)} F$ for a given graph: it may fail, in certain cases, because of some obstruction not revealed by the study of the set $\left\{n_{i}\right\}$ alone. This is what has been announced by Ocneanu [33] for one of the graphs of $\widehat{s l}(3)$ listed in [2].

\section{Generalised twisted boundary conditions}

\subsection{Twist operators}

In the discussion of sect 1.2 , we computed the partition function on a torus by identifying the states at the two ends of a cylinder through the trace operation. Let us now imagine that we allow the action of a (non-local) operator $X$, attached to a cycle of the cylinder, before identifying these two ends, and thus compute $Z_{X}=\operatorname{tr}_{\mathcal{H}_{P}} X e^{2 \pi i\left[\tilde{\tau}\left(L_{0}-c / 24\right)-\tilde{\tau}^{*}\left(\bar{L}_{0}-c / 24\right)\right]}$. The effect of this operator is to "twist" the b.c. In statistical mechanics, this is a familiar operation, often referred to as a "seam". A typical example is offered by the Ising model, where antiperiodic b.c. may be imposed on the spins in the "time" direction by inserting the operator $X$ which flips the spins along a generatrix of the cylinder. The $X$ are not arbitrary: we demand that they commute with the action of the two copies of the Virasoro algebra

$$
\left[L_{n}, X\right]=\left[\bar{L}_{n}, X\right]=0 .
$$



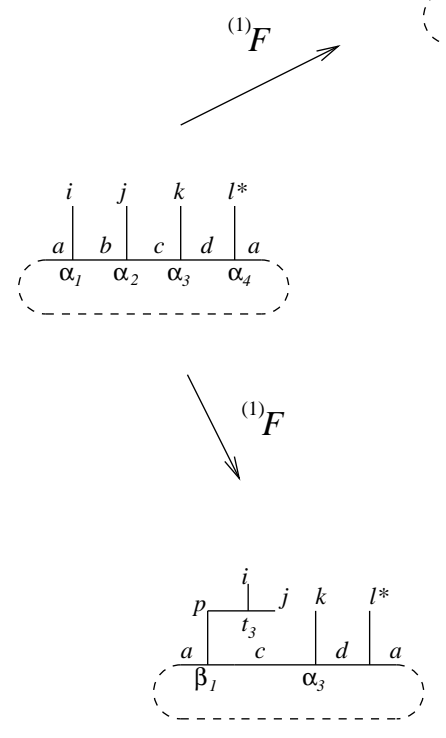
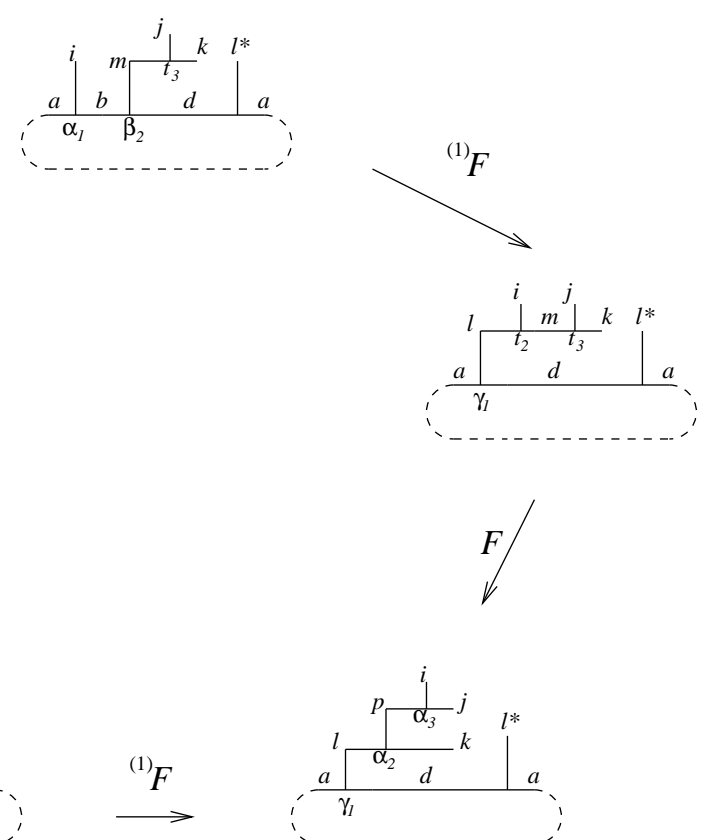

Fig. 3: The mixed pentagon identity expressing the associativity of the fusion of boundary fields.

As the $L_{n}, \bar{L}_{n}$ generate infinitesimal diffeomorphisms, this condition may be regarded as expressing that the operators $X$ are invariant under an arbitrary deformation of the closed line to which they are attached. Returning to our example of the lattice Ising model, the antiperiodicity condition on the spins may be imposed along any non contractible, non self-intersecting curve around the cylinder, the deformation of which is made possible by the invariance of the model under local changes of the spin variable $\sigma_{i} \rightarrow-\sigma_{i}$. In general, when the CFT has a chiral algebra larger than Vir, we impose conditions of commutation of $X$ with all the generators of that algebra.

Now, the solutions of (4.1) are readily found, through a line of argument parallel to that of sect. 2.2 and a new application of Schur's lemma. $X$ acts in $\mathcal{H}_{P}$ (see eq (1.15)) and is in general a linear combination of solutions of (4.1) mapping some $\mathcal{V}_{j} \otimes \overline{\mathcal{V}_{\bar{j}}}$ into $\mathcal{V}_{j^{\prime}} \otimes \overline{\mathcal{V}_{\overline{j^{\prime}}}}$. Irreducibility of the $\mathcal{V}_{\text {s tells us }}$ that $X$ is non trivial only if $j=j^{\prime}$ and $\bar{j}=\bar{j}^{\prime}$. If the multiplicity $Z_{j \bar{j}}=1$, $X$ is then proportional to the projector in $\mathcal{V}_{j} \otimes \overline{\mathcal{V}_{\bar{j}}}$. If $Z_{j \bar{j}}>1$, however, $X$ is a linear superposition of operators intertwining the different copies of $\mathcal{V}_{j} \otimes \overline{\mathcal{V}_{\bar{j}}}$ 
and acting as a projector in each

$$
P^{\left(j, \bar{j} ; \alpha, \alpha^{\prime}\right)}:\left(\mathcal{V}_{j} \otimes \overline{\mathcal{V}}_{\bar{j}}\right)^{\left(\alpha^{\prime}\right)} \rightarrow\left(\mathcal{V}_{j} \otimes \overline{\mathcal{V}}_{\bar{j}}\right)^{(\alpha)} \quad \alpha, \alpha^{\prime}=1, \cdots, Z_{j \bar{j}}
$$

In other words, if $|j, \mathbf{n}\rangle \otimes|\bar{j}, \overline{\mathbf{n}}\rangle$ is an orthonormal basis of $\mathcal{V}_{j} \otimes \overline{\mathcal{V}_{\bar{j}}}$, with $\mathbf{n}, \overline{\mathbf{n}}$ multi-indices labelling the descendent states,

$$
P^{\left(j, \bar{j} ; \alpha, \alpha^{\prime}\right)}=\sum_{\mathbf{n}, \overline{\mathbf{n}}}(|j, \mathbf{n}\rangle \otimes|\bar{j}, \overline{\mathbf{n}}\rangle)^{(\alpha)}(\langle j, \mathbf{n}| \otimes\langle\bar{j}, \overline{\mathbf{n}}|)^{\left(\alpha^{\prime}\right)} \quad \alpha, \alpha^{\prime}=1, \cdots Z_{j \bar{j}}
$$

There are thus $\sum_{j, \bar{j}}\left(Z_{j \bar{j}}\right)^{2}$ linearly independent operators $P^{\left(j, \bar{j} ; \alpha, \alpha^{\prime}\right)}$, which play in this problem the rôle of the Ishibashi states in the discussion of sect 2 . They may be composed according to

$$
P^{\left(j_{1}, \bar{j}_{1} ; \alpha_{1}, \alpha_{1}^{\prime}\right)} P^{\left(j_{2}, \bar{j}_{2} ; \alpha_{2}, \alpha_{2}^{\prime}\right)}=\delta_{j_{1} j_{2}} \delta_{\bar{j}_{1} \bar{j}_{2}} \delta_{\alpha_{1}^{\prime} \alpha_{2}} P^{\left(j_{1}, \bar{j}_{1} ; \alpha_{1}, \alpha_{2}^{\prime}\right)}
$$

The most general solution $X$ of (4.1) may then be written as

$$
X_{x}=\sum_{j \bar{j}, \alpha, \alpha^{\prime}} \frac{\Psi_{x}^{\left(j, \bar{j} ; \alpha, \alpha^{\prime}\right)}}{\sqrt{S_{1 j} S_{1 \bar{j}}}} P^{\left(j, \bar{j} ; \alpha, \alpha^{\prime}\right)}
$$

where the $\Psi$ are complex numbers. The denominator $\sqrt{S_{1 j} S_{1 \bar{j}}}$ is introduced for later convenience. We shall denote by $\widetilde{\mathcal{V}}$ the set of labels $x$ and use the label $x=1$ for the identity operator

$$
X_{1}:=\operatorname{Id}=\sum_{j \bar{j}, \alpha} P^{(j, \bar{j} ; \alpha, \alpha)},
$$

for which

$$
\Psi_{1}^{\left(j, \bar{j} ; \alpha, \alpha^{\prime}\right)}=\sqrt{S_{1 j} S_{1 \bar{j}}} \delta_{\alpha \alpha^{\prime}}=: \Psi_{1}^{(j, \bar{j})} \delta_{\alpha \alpha^{\prime}}
$$

Using (4.4) and the hermitian conjugation properties of the projectors

$$
\left(P^{\left(j, \bar{j} ; \alpha, \alpha^{\prime}\right)}\right)^{\dagger}=P^{\left(j, \bar{j} ; \alpha^{\prime}, \alpha\right)}
$$

we may compose two such $X$ as

$$
X_{x}^{\dagger} X_{y}=\sum_{j, \bar{j}, \alpha, \alpha^{\prime}, \alpha^{\prime \prime}} \frac{\Psi_{x}^{\left(j, \bar{j} ; \alpha, \alpha^{\prime}\right) *} \Psi_{y}^{\left(j, \bar{j} ; \alpha^{\prime \prime}, \alpha^{\prime}\right)}}{S_{1 j} S_{1 \bar{j}}} P^{\left(j, \bar{j} ; \alpha, \alpha^{\prime \prime}\right)}
$$

Insertion of one or two such $X$ will be sufficient to expose their most interesting features. 


\subsection{A new consistency condition}

We may now repeat the steps followed in sect 2 . We consider a finite cylinder of length $2 L$ and of circumference $T$. If $\tilde{q}=\exp 2 i \pi \tilde{\tau}, \tilde{\tau}=2 i L / T$, we have

$$
\operatorname{tr}_{\mathcal{H}_{P}}\left(P^{\left(j, \bar{j} ; \alpha, \alpha^{\prime}\right)} \tilde{q}^{L_{0}-c / 24} \tilde{q}^{\bar{L}_{0}-c / 24}\right)=\chi_{j}(\tilde{q}) \chi_{\bar{j}}(\tilde{q}) \delta_{\alpha \alpha^{\prime}},
$$

and the partition function on that cylinder in the presence of two twists reads

$$
Z_{x \mid y}:=Z_{X_{x}^{\dagger} X_{y}}=\sum_{\substack{j, \bar{j} \in \mathcal{I} \\ \alpha, \alpha^{\prime}=1, \cdots, Z_{j \bar{j}}}} \frac{\Psi_{x}^{\left(j, \bar{j} ; \alpha, \alpha^{\prime}\right) *} \Psi_{y}^{\left(j, \bar{j} ; \alpha, \alpha^{\prime}\right)}}{S_{1 j} S_{1 \bar{j}}} \chi_{j}(\tilde{q}) \chi_{\bar{j}}(\tilde{q}) .
$$

In particular, for $x=y=1$, we find

$$
Z_{1 \mid 1}=\sum_{j, \bar{j}, \alpha} \chi_{j}(\tilde{q}) \chi_{\bar{j}}(\tilde{q})=\sum_{j, \bar{j} \in \mathcal{I}} Z_{j \bar{j}} \chi_{j}(\tilde{q}) \chi_{\bar{j}}(\tilde{q})
$$

which is the modular invariant partition function describing the system with no twist.

As the commutation relation (4.1) guarantees the existence of a well defined stress-energy tensor on the cylinder consistent with the identification of its two ends, we may also carry out the computation of $Z_{x \mid y}$ in another way, by making use of the Hamiltonian corresponding to the $\Re e w$-translation operator. Then the partition function $Z_{x \mid y}$ is obtained as the trace of the corresponding evolution operator in a Hilbert space

$$
\mathcal{H}_{x \mid y}=\oplus_{i, \bar{i} \in \mathcal{I}} \tilde{V}_{i \bar{i}^{*} ; x}{ }^{y} \mathcal{V}_{i} \otimes \overline{\mathcal{V}}_{\bar{i}}
$$

where the non negative integer multiplicities $\tilde{V}_{i \bar{i} ; x}{ }^{y}$ depend on the twists $x$ and $y$. In the trivial case $x=y=1$, they must reduce to

$$
\tilde{V}_{i \bar{\imath}^{*} ; 1}{ }^{1}=Z_{i \bar{i}} .
$$

We can thus complete the calculation as in the absence of the $X$ operator(s) and get, with $q=\exp 2 \pi i \tau, \tau=-1 / \tilde{\tau}=i T / 2 L$

$$
\begin{aligned}
Z_{x \mid y} & =\operatorname{tr}_{\mathcal{H}_{x \mid y}} q^{L_{0}-c / 24} q^{\bar{L}_{0}-c / 24} \\
& =\sum_{i, \bar{i} \in \mathcal{I}} \tilde{V}_{i \bar{i} ; x^{y}} \chi_{i}(q) \chi_{\bar{i}}(q) .
\end{aligned}
$$


Identifying the two expressions (4.11) and (4.15) after a modular transformation of the characters, we get

$$
\tilde{V}_{i \bar{i} ; x}{ }^{y}=\sum_{j, \bar{j}, \alpha, \alpha^{\prime}} \frac{S_{i j} S_{\bar{i} \bar{j}}}{S_{1 j} S_{1 \bar{j}}} \Psi_{x}^{\left(j, \bar{j} ; \alpha, \alpha^{\prime}\right)} \Psi_{y}^{\left(j, \bar{j} ; \alpha, \alpha^{\prime}\right) *}, \quad i, \bar{i} \in \mathcal{I} .
$$

In fact, as in sect 2 , there is an ambiguity inherent to the use of specialised characters. See [40] for a discussion of that point. The similarity of condition (4.16) with Cardy's equation in the case of open boundaries is quite striking. We shall in fact exploit equation (4.16) in a way parallel to what we did in sect 2 .

To proceed, we make the additional assumption that the $\Psi_{x}^{\left(j, \bar{j} ; \alpha, \alpha^{\prime}\right)}$ form a unitary (i.e. orthonormal and complete) change of basis from the $P^{\left(j, \bar{j}, \alpha, \alpha^{\prime}\right)}$ to the $X_{x}$ operators. This implies that the set $\widetilde{\mathcal{V}}$ of labels $x$ has a cardinality equal to $\sum\left(Z_{j \bar{j}}\right)^{2}$. The integer numbers $\tilde{V}_{i \bar{i} ; x}{ }^{y}$ will be considered either as the entries of $|\mathcal{I}| \times|\mathcal{I}|$ matrices $\tilde{V}_{x}^{y}, x, y \in \widetilde{\mathcal{V}}$, or as those of $|\widetilde{\mathcal{V}}| \times|\widetilde{\mathcal{V}}|$ matrices $\tilde{V}_{i \bar{i}}$, $i, \bar{i} \in \mathcal{I}$.

Following the same argument as in sect 2.4, equation (4.16) may be regarded as the spectral decomposition of the matrices $\tilde{V}_{i \bar{i}}$ into their orthogonal eigenvectors $\Psi$ and eigenvalues $S_{i j} S_{\bar{i} \bar{j}} / S_{1 j} S_{1 \bar{j}}$. As the latter form a representation of the tensor product of two copies of Verlinde fusion algebra, the same holds true for the $\tilde{V}$ matrices:

$$
\tilde{V}_{i_{1} j_{1}} \tilde{V}_{i_{2} j_{2}}=\sum_{i_{3}, j_{3}} \mathcal{N}_{i_{1} i_{2}}{ }^{{ }^{3}} \mathcal{N}_{j_{1} j_{2}}{ }^{j_{3}} \tilde{V}_{i_{3} j_{3}}
$$

Combining (4.14) with (4.17), we have in particular

$$
\sum_{i_{3} j_{3}} \mathcal{N}_{i_{1} i_{2}}{ }^{i_{3}} \mathcal{N}_{j_{1} j_{2}}^{{ }^{j_{3}}} Z_{i_{3} j_{3}}=\sum_{x} \tilde{V}_{i_{1} j_{1}^{*} ; 1}{ }^{x} \tilde{V}_{i_{2} j_{2}^{*} ; x}{ }^{1}
$$

which is the way the matrices $\tilde{V}_{i j ; 1}{ }^{x}=\tilde{V}_{i^{*} j^{*} ; x}{ }^{1}$ appeared originally in the work of Ocneanu. As will be explained below, all $\tilde{V}_{x}^{y}$ may be reconstructed from the simpler Ocneanu matrices $\tilde{V}_{1}{ }^{x}$. According to an argument already used for the $n$ matrices, by (4.17), it is sufficient to specify $\tilde{V}_{f 1}$ and $\tilde{V}_{1 f}$ for a generating subset of representations $f \in \mathcal{I}$ to determine the whole set of matrices $\tilde{V}_{i j}$; for example $f=2$ in the case of $s l(2)$ theories. The matrices $\tilde{V}_{f 1}$ and $\tilde{V}_{1 f}$ may be regarded as the adjacency matrices of graphs with the common set of vertices $\widetilde{\mathcal{V}}$. It is convenient to draw these graphs on the same chart, with edges of 


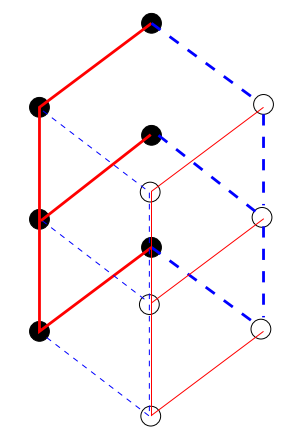

Fig. 4: The Ocneanu graph of type $E_{6}$ : the edges corresponding to $\tilde{V}_{21}$, resp. $\tilde{V}_{12}$, have been drawn in full red, resp. blue broken

different colours, and we refer to the resulting multiple graph $\tilde{G}$ as Ocneanu graph.

In diagonal cases, the matrices $\tilde{V}_{i j}$ are labelled by the set $\widetilde{\mathcal{V}}=\mathcal{I}$ of representation labels and must satisfy (4.17) and (4.14). They are simply

$$
\tilde{V}_{i j}=\mathcal{N}_{i} \mathcal{N}_{j}
$$

i.e. $\tilde{V}_{i j ; x^{y}}=\sum_{k} Z_{i x}{ }^{k} Z_{k j}{ }^{y}$, and the Ocneanu graph is simply made of two identical copies of the fusion graph of $\mathcal{N}_{f}$ with their vertices identified. The example of the Ocneanu graph for the $\widehat{s l}(2)$ theory of type $E_{6}$ is presented on Fig. 4. More graphs and more details on these graphs and their construction may be found in $[37,33,41,42,43,39]$.

\subsection{The fusion algebra of defect lines}

We may finally combine the two situations encountered in the discussion of these lectures and consider the insertion of twist operators in the presence of open boundaries with boundary states $|a\rangle$ and $\langle b|$. It is a simple exercise left to the reader to check that

$$
Z_{a x}^{b}:=\left\langle b\left|X_{x}^{\dagger} \tilde{q}^{L_{0}-\frac{c}{24}} \tilde{q}^{\bar{L}_{0}-\frac{c}{24}}\right| a\right\rangle=\sum_{i}\left(n_{i} \tilde{n}_{x}\right)_{a^{*}}^{b^{*}} \chi_{i}(q),
$$

with $\tilde{n}_{x}=\left\{\tilde{n}_{a x}^{b}\right\}$ a new set of multiplicities, i.e. non negative integers,

$$
\tilde{n}_{a x}{ }^{b}=\sum_{j, \alpha, \beta} \psi_{a}^{(j, \alpha)} \frac{\Psi_{x}^{(j, j ; \alpha, \beta)}}{\Psi_{1}^{(j, j)}} \psi_{b}^{(j, \beta) *} .
$$


These new matrices $\tilde{n}_{x}$ play a rôle parallel to that of the $n_{i}$, and indeed, they form themselves a (non-negative integer valued matrix) representation of an algebra with (non negative integer) structure constants

$$
\begin{gathered}
\tilde{n}_{x} \tilde{n}_{y}=\sum_{z} \widetilde{N}_{x y}{ }^{z} \tilde{n}_{z} \\
\widetilde{N}_{x y}{ }^{z}=\sum_{j, \bar{j} ; \alpha} \sum_{\beta, \gamma} \Psi_{x}^{(j, \bar{j} ; \alpha, \beta)} \frac{\Psi_{y}^{(j, \bar{j} ; \beta, \gamma)}}{\Psi_{1}^{(j, \bar{j})}} \Psi_{z}^{(j, \bar{j} ; \alpha, \gamma) *} .
\end{gathered}
$$

One also easily checks that

$$
Z_{y \mid z}=\sum_{x} \tilde{N}_{y x}^{z} Z_{1 \mid x}
$$

or equivalently, $\tilde{V}_{y}^{z}=\sum_{x} \tilde{N}_{y x}^{z} \tilde{V}_{1}^{x}$, as announced above. The matrices $\tilde{N}_{x}:=$ $\left\{\tilde{N}_{y x}{ }^{z}\right\}$ form an associative algebra

$$
\widetilde{N}_{x} \widetilde{N}_{y}=\sum_{z} \tilde{N}_{x y}^{z} \tilde{N}_{z}
$$

which may be called the "fusion algebra of defect lines".

There is, however, a major and most intriguing new feature which distinguishes this new algebra $\widetilde{N}$ from the standard fusion algebra $N_{i}$. In general, if some of the multipicities $Z_{j \bar{j}}>1$, this new algebra is non commutative! [37]. Physically, this must be interpreted as the lack of commutativity of the twist operators (or of the defect lines to which they are attached).

We conclude this section with the remark that the Ocneanu graphs $\tilde{G}$ contain an information on the OPA of fields with arbitrary spins, generalising the relation of the scalar fields OPE coefficients to the Pasquier algebra discussed above, see [39] for details.

\section{The underlying quantum algebra}

The various sets of multiplicities that we have encountered $\left\{\mathcal{N}_{i}\right\},\left\{n_{i}\right\},\left\{\widetilde{N}_{x}\right\}$ and $\left\{\tilde{n}_{x}\right\}$, and the inter-relations that they satisfy, are in fact the indicators of a deep and beautiful structure. In the combinatorial approach of $[37,44]$, one constructs a simplicial complex out of the following elements (Fig 5): 2 kinds of 0 -simplices, indicated by black or white dots, 3 kinds of oriented edges, 4 kinds of 2 -faces and 5 kinds of tetrahedral 3 -simplices. The triangular faces come 


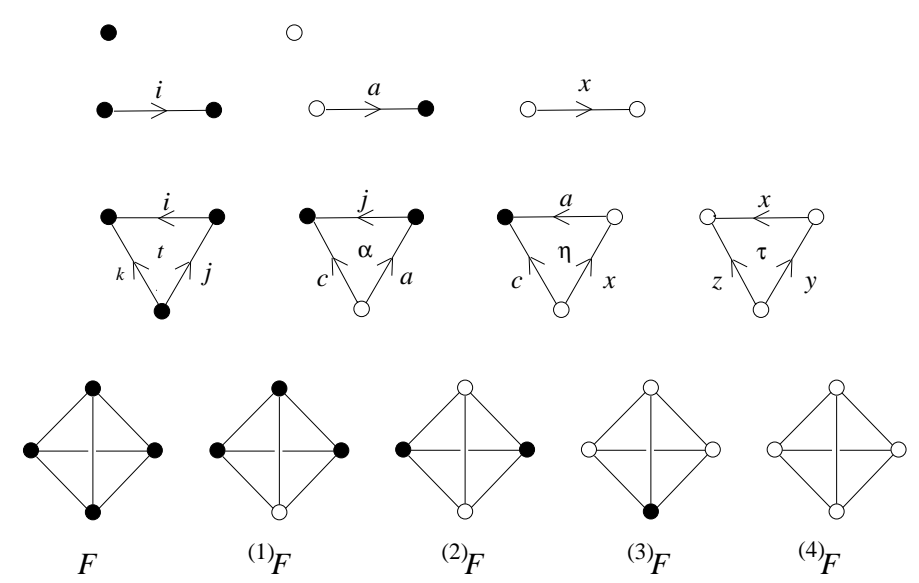

Fig. 5: The simplices

with a multiplicity index, $t=1, \cdots, \mathcal{N}_{i j}{ }^{k}, \alpha=1, \cdots, n_{i a}{ }^{c}, \eta=1, \cdots, \tilde{n}_{a x}{ }^{c}$, $\tau=1, \cdots, \widetilde{N}_{x y}{ }^{z}$, and these multiplicities obey the set of relations

$$
\begin{aligned}
\sum_{\ell \in \mathcal{I}} \mathcal{N}_{i m}{ }^{\ell} \mathcal{N}_{j \ell}{ }^{n} & =\sum_{k \in \mathcal{I}} \mathcal{N}_{i j}{ }^{k} \mathcal{N}_{k m}{ }^{n} \\
\sum_{c \in \mathcal{V}} n_{i a}{ }^{c} n_{j c}{ }^{b} & =\sum_{k \in \mathcal{I}} \mathcal{N}_{i j}{ }^{k} n_{k a}{ }^{b} \\
\sum_{x \in \widetilde{\mathcal{V}}} \tilde{n}_{a x}{ }^{\prime} \tilde{n}_{x^{*} b^{\prime}}{ }^{b} & =\sum_{i \in \mathcal{I}} n_{i a}{ }^{b} n_{i^{*} b^{\prime}}{ }^{\prime} \\
\sum_{c \in \mathcal{V}} \tilde{n}_{a x}{ }^{c} \tilde{n}_{c y}{ }^{b} & =\sum_{z \in \widetilde{\mathcal{V}}} \widetilde{N}_{x y}{ }^{z} \tilde{n}_{a z}{ }^{b} \\
\sum_{v \in \widetilde{\mathcal{V}}} \widetilde{N}_{w x}{ }^{v} \widetilde{N}_{v y}{ }^{u} & =\sum_{z \in \widetilde{\mathcal{V}}} \widetilde{N}_{x y}{ }^{z} \widetilde{N}_{w z}{ }^{u} .
\end{aligned}
$$

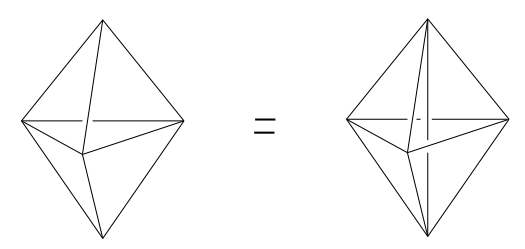

Fig. 6: Pentagonal identity resulting from two ways of cutting a solid into tetrahedra 
With each tetrahedron, we want to attach a complex number, (a 3-chain), $F_{m n}\left[\begin{array}{ll}i & j \\ k & l\end{array}\right]_{t t^{\prime}}^{u u^{\prime}},{ }^{(1)} F_{b k}\left[\begin{array}{ll}i & j \\ c & a\end{array}\right]_{\alpha \alpha^{\prime}}^{\beta t},{ }^{(2)} F_{i x}\left[\begin{array}{ll}a & b \\ c & d\end{array}\right]_{\alpha \beta}^{\eta \zeta},{ }^{(3)} F_{b z}\left[\begin{array}{ll}x & y \\ c & a\end{array}\right]_{\eta \eta^{\prime}}^{\zeta \tau},{ }^{(4)} F_{u v}\left[\begin{array}{ll}x & y \\ w & z\end{array}\right]_{\tau \tau^{\prime}}^{v v^{\prime}}$, which must satisfy all the pentagon identities resulting from the decorations with black and white colors of the vertices of the solid of Fig 6 , and from its cutting either into three or into two tetrahedra. These identities include those already mentioned in sect 1.1 and 3 . As in these previous encounters, they realise unitary (with the exception of the invertible ${ }^{(2)} F$ ) changes of basis, and the five relations above are necessary conditions for each pair of bases, (depicted by double triangles with a fixed $0,1,2,3,4$ number of white vertices respectively) to have the same dimension.

These data, if they exist, enable one to construct an abstract algebra, the Ocneanu "double triangle algebra" [33], which appears as the quantum symmetry of the CFT [39]. Being defined in terms of the $n$ 's etc, this algebra is intrinsic to the CFT, its spectrum of bulk and boundary states etc. We refer the reader to $[33,44,43,39]$ for a thorough discussion of its definition, its various interpretations, and for a fairly extensive list of references.

The algebra $\mathcal{A}$ is constructed as follows. One first considers a basis $\left|e_{b a}^{j, \alpha}\right\rangle$ labelled and represented pictorially in the same way as the boundary fields of sect 3 , or by the triangles with one white vertex in Fig. 6. One defines tensor products of two such vectors provided the intermediate labels coincide, and the matrix elements of ${ }^{(1)} F$ act as " $3 j$-symbols", while the $F$ are the recoupling, or $6 j-$, symbols. The algebra itself is generated by the matrix units $\left|e_{c a}^{j, \beta}\right\rangle\left\langle e_{c^{\prime}, a^{\prime}}^{j, \beta^{\prime}}\right|$. One shows that this algebra has a product and a coassociative coproduct (inherited from the previous tensor product), a counit and an antipode, but that the axioms of Hopf algebras are not all satisfied: $\mathcal{A}$ is a "weak Hopf algebra", also called a "quantum groupoid" [44]. Its dual has a basis labelled by indices $x$ as above, relation (5.1) guarantees the existence of an invertible change ${ }^{(2)} F$ from the original basis to the dual one. In the dual, the matrices ${ }^{(3)} F$ and ${ }^{(4)} F$ play the rôles of $3 j$ - and $6 j$-symbols. The dimension of $\mathcal{A}$ is given by the number of all double triangles with two black and two white vertices, i.e., by the sum over $a, a^{\prime}, b, b^{\prime}$ of each side of (5.1).

\section{Integrable lattice models}

It should be recalled that in parallel to the conformal field theoretic discussion sketched in these notes, one may study a class of lattice integrable models, the so-called face, or height, or RSOS, models, which are also described in terms of the same graphs. There the degrees of freedom are attached to sites of a square lattice, and are assigned to take their value in the set of vertices of the chosen graph. Typically, in the simplest models, neighbouring sites 
on the lattice are assigned neighbouring vertices on the graph. Boltzmann weights are given for each configuration of four vertices around a square face. They depend on an additional parameter, the spectral parameter $u$, and must satisfy an integrability condition, the celebrated Yang-Baxter equation. This is realised algebraically through a representation of the Temperley-Lieb algebra, or of some other quotient of the Hecke algebra, constructed on the graph as follows. For a triplet of sites $\mathbf{n}-1, \mathbf{n}, \mathbf{n}+1$ along a diagonal zig-zag line on the lattice, and "heights" $a, b$ or $d$ and $c$ assigned to them, the face Boltzmann weight reads

$$
X_{\mathbf{n}}(u)=\sum_{\mathbf{n}-1}^{a} \int_{b^{\gamma}}^{d} \sum_{\mathbf{n}+1}^{\gamma} c=\sin \left(\frac{\pi}{h}-u\right) \delta_{b d}+\sin u\left(U_{\mathbf{n}}\right)_{b d}
$$

The $U$ are the generators of the Hecke algebra, i.e. satisfy

$$
\begin{aligned}
U_{\mathbf{n}}^{2} & =2 \cos \frac{\pi}{h} U_{\mathbf{n}} \\
U_{\mathbf{n}} U_{\mathbf{n}+1} U_{\mathbf{n}}-U_{\mathbf{n}} & =U_{\mathbf{n}+1} U_{\mathbf{n}} U_{\mathbf{n}+1}-U_{\mathbf{n}+1} \\
U_{\mathbf{n}} U_{\mathbf{m}} & =U_{\mathbf{m}} U_{\mathbf{n}} \quad \text { if }|\mathbf{n}-\mathbf{m}| \geq 2 .
\end{aligned}
$$

As a consequence, the face weights satisfy the Yang-Baxter identity

$$
X_{\mathbf{n}}(u) X_{\mathbf{n}+1}(u+v) X_{\mathbf{n}}(v)=X_{\mathbf{n}+1}(v) X_{\mathbf{n}}(u+v) X_{\mathbf{n}+1}(u) .
$$

The Pasquier models [45] give the simplest and most explicit example: the relevant graphs are once again the ADE diagrams, and the $U$ matrix element reads

$$
\left(U_{\mathbf{n}}\right)_{b d}=\delta_{a c} \frac{\sqrt{\psi_{b}^{1} \psi_{d}^{1}}}{\psi_{a}^{1}} .
$$

They are related to the $s l(2)$ algebra. Although generalisations to higher rank are known to exist, there is a lack of such explicit and general formulas. In general, there are additional edge degrees of freedom $\alpha, \gamma, \alpha^{\prime}, \gamma^{\prime}, \alpha=1, \cdots, n_{\square a}{ }^{b}$, etc. The weights for the (generalised) $A$-type graphs are known from the work of Jimbo et al, and Wenzl [46]. In [26,47] explicit expressions have been given for models associated with some of the graphs of $\widehat{s l}(3)$, and a general result has been obtained in [41] for graphs of $\widehat{s l}(N)$ corresponding to conformal embeddings. The recent observation by Ocneanu [33] that one may write the above generator of the Hecke algebra as

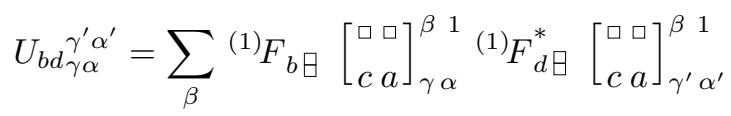


in terms of the ${ }^{(1)} F$ matrix previously introduced is thus a significant progress, both practically and conceptually, since it connects problems of apparently different nature.

Finally, in these lattice models, it is legitimate to wonder if boundaries may be introduced without spoiling integrability. This requires a careful determination of the boundary Boltzmann weights, satisfying the so-called Boundary Yang-Baxter Equation [3]. This has now been completed for the unitary minimal models: a large class of boundary weights has been found, which at criticality, match perfectly what we have learnt from BCFT [31]. It remains to connect these boundary weights with quantities defined previously in the context of BCFT to have a fully consistent and unified picture of all questions of boundary conditions in integrable lattice models and conformal theories.

\section{Acknowledgements}

J.-B. Z. is happy to thank Professors Z. Horvath and L. Palla, the Eötvös University and Bolyai College for their invitation and hospitality in the wonderful city of Budapest and all the participants of the school and conference for providing a stimulating atmosphere. A good part of the work presented here results from several enjoyable collaborations with R. Behrend and P. Pearce. 


\section{References}

[1] H. Saleur, Lectures on Non Perturbative Field Theory and Quantum Impurity Problems, I and II, cond-mat 9812110, cond-mat 0007309.

[2] R.E. Behrend, P.A. Pearce, V.B. Petkova and J.-B. Zuber, Nucl. Phys. B 579 (2000) 707-773, hep-th 9908036.

[3] R.E. Behrend and P.A. Pearce, J. Phys. A 29 (1996) 7827-7835; Int. J. Mod. Phys. 11 (1997) 2833-2847.

[4] P. Dorey, lectures at this school.

[5] K. Graham, I. Runkel and G.M.T. Watts, PRHEP-tmr2000/040 (Proceedings of the TMR network conference Nonperturbative Quantum Effects 2000), hep-th 0010082.

[6] V. Kac, Infinite dimensional algebras, Cambridge University P.; V.G. Kac and D.H. Peterson, Adv. Math. 53 (1984) 125-264;

J. Fuchs, Affine Lie Algebras and Quantum Groups, Cambridge Univ. Pr. 1992.

[7] P. Goddard, A. Kent and D. Olive, Comm. Math. Phys. 103 (1986) 105-119.

[8] P. Di Francesco, P. Mathieu and D. Sénéchal, Conformal Field Theory, Springer Verlag 1997.

[9] E. Verlinde, Nucl. Phys. B300 [FS22] (1988) 360-376.

[10] G. Moore and N. Seiberg, Comm. Math. Phys. 123 (1989) 177-254; Lectures on RCFT, Physics, Geometry and Topology, Plenum Press, New York, 1990.

[11] A. Cappelli, C. Itzykson and J.-B. Zuber, Nucl. Phys. B280 [FS18] (1987) 445465; Comm. Math. Phys. 113 (1987) 1-26;

A. Kato, Mod. Phys. Lett. A2 (1987) 585-600.

[12] T. Gannon, Comm. Math. Phys. 161 (1994) 233-263; The Classification of SU(3) Modular Invariants Revisited, hep-th 9404185.

[13] C. Itzykson, Nucl. Phys. (Proc. Suppl.) 5B (1988) 150-165 ;

P. Degiovanni, Comm. Math. Phys. 127 (1990) 71-99.

[14] T. Gannon, The monstruous moonshine and the classification of CFT, hep-th 9906167.

[15] J.-B. Zuber, CFT, BCFT, ADE and all that, to appear in the proceedings of the Bariloche Summer School, "Quantum Symmetries in Theoretical Physics and Mathematics", Jan 2000, eds R. Coquereaux, A. Garcia and R. Trinchero, hep-th 0006151.

[16] Vl.S. Dotsenko and V.A. Fateev, Nucl. Phys. B 251 [FS13] (1985) 691-734.

[17] A.B. Zamolodchikov and V.A. Fateev, Sov. J. Nucl.Phys. 43 (1986) 657-664. 
[18] V.B. Petkova and J.-B. Zuber, Nucl. Phys. B 438 (1995) 347-372, hep-th 9410209.

[19] V. Pasquier, J. Phys. A20 (1987) 5707-5717.

[20] G. Watts, private communication.

[21] J. Fuchs and C. Schweigert, Nucl. Phys. B 530 (1998) 99-136, hep-th 9712257.

[22] A. Recknagel and V. Schomerus, Nucl. Phys. B 531 (1998) 185-225.

[23] J.L. Cardy, Nucl. Phys. 324 (1989) 581-596.

[24] L. Chim, Int. J. Mod. Phys. A11 (1996) 4491-4512, hep-th 9510008; I. Affleck, Edge critical behaviour of the 2-dimensional tri-critical Ising model, cond-mat 0005286.

[25] A. Recknagel, D. Roggenkamp, V. Schomerus, On relevant boundary perturbations of unitary minimal models, hep-th 0003110.

[26] P. Di Francesco and J.-B. Zuber, Nucl. Phys. B338 (1990) 602-646.

[27] G. Pradisi, A. Sagnotti and Ya.S. Stanev, Phys. Lett. 381 (1996) 97-104.

[28] F.M. Goodman, P. de la Harpe and V.F.R. Jones, Coxeter Graphs and Towers of Algebras, Springer-Verlag, Berlin (1989).

[29] I. Affleck, M. Oshikawa and H. Saleur, J. Phys A 31 (1998) 5827-5842, cond-mat 9804117.

[30] R.E. Behrend, P.A. Pearce and J.-B. Zuber, J.Phys. A31 (1998) L763-L770, hep-th 9807142.

[31] R.E. Behrend and P.A. Pearce, Integrable and Conformal Boundary Conditions for $\widehat{s l}(2) A-D-E$ Lattice Models and Unitary Conformal Field Theories, hep-th 0006094, J. Stat Phys. to appear.

[32] P. Di Francesco and J.-B. Zuber, in Recent Developments in Conformal Field Theories, Trieste Conference 1989, S. Randjbar-Daemi, E. Sezgin and J.-B. Zuber eds., World Scientific 1990 ;

P. Di Francesco, Int. J. Mod. Phys. A7 (1992) 407-500.

[33] A. Ocneanu, lectures at the school "Quantum Symmetries in Theoretical Physics and Mathematics", January 2000, Bariloche, R. Coquereaux, A. Garcia and R. Trinchero eds, to appear.

[34] J.L. Cardy and D.C. Lewellen, Phys. Lett. B 259 (1991) 274-278;

D.C. Lewellen, Nucl. Phys. B 372 (1992) 654-682.

[35] I. Runkel, Nucl. Phys. B 549 (1999) 563-578, hep-th 9811178.

[36] I. Runkel, Nucl. Phys. B 579 (2000) 561-589, hep-th 9908046.

[37] A. Ocneanu, Paths on Coxeter Diagrams, in Lectures on Operator Theory Fields Institute Monographies, Rajarama Bhat et al edrs, AMS 1999. 
[38] V.B. Petkova and J.-B. Zuber, PRHEP-tmr2000/038 (Proceedings of the TMR network conference Nonperturbative Quantum Effects 2000), hep-th 0009219.

[39] V.B. Petkova and J.-B. Zuber, The many faces of Ocneanu cells, hep-th 0101151.

[40] V.B. Petkova and J.-B. Zuber, Generalised twisted partition functions, Phys. Lett. $B$ to appear, hep-th 0011021.

[41] F. Xu, Comm. Math. Phys. 192 (1998) 349-403.

[42] J. Böckenhauer and D.E. Evans, Comm.Math. Phys. 197 (1998) 361-386; ibidem 200 (1999) 57-103, hep-th 9805023; ibidem 205 (1999) 183-228, hep-th 9812110.

[43] J. Böckenhauer, D.E. Evans, and Y. Kawahigashi, Comm.Math. Phys. 208 (1999) 429-487, math-oa 9904109; ibidem 210 (2000) 733-784.

[44] G. Böhm and K. Szlachányi, Lett. Math. Phys. 200 (1996) 437-456, q-alg 9509008 ;

G. Böhm, Weak $C^{*}$-Hopf Algebras and their Application to Spin Models, PhD Thesis, Budapest 1997.

[45] V. Pasquier, Nucl. Phys. B285 [FS19] (1987) 162-172.

[46] M. Jimbo, T. Miwa and M. Okado, Lett. Math. Phys. 14 (1987) 123-131; Comm. Math. Phys. 119 (1988) 543-565;

H. Wenzl, Inv. Math. 92 (1988) 349.

[47] N. Sochen, Nucl. Phys. B360 (1991) 613-640. 